Ann. Scient. Éc. Norm. Sup.,

$4^{\mathrm{e}}$ série, t. 39, 2006, p. 649 à 677.

\title{
SMOOTHNESS OF ITÔ MAPS AND DIFFUSION PROCESSES ON PATH SPACES (I)
}

\author{
BY XIANG-DONG LI AND TERRY J. LYONS
}

ABStRACT. - Let $p \in[1,2)$ and $\alpha, \varepsilon>0$ be such that $\alpha \in(p-1,1-\varepsilon)$. Let $V, W$ be two Euclidean spaces. Let $\Omega_{p}(V)$ be the space of continuous paths taking values in $V$ and with finite $p$-variation. Let $k \in \mathbb{N}$ and $f: W \rightarrow \operatorname{Hom}(V, W)$ be a $\operatorname{Lip}(k+\alpha+\varepsilon)$ map in the sense of E.M. Stein [Stein E.M., Singular integrals and differentiability properties of functions, Princeton Mathematical Series, vol. 30, Princeton University Press, Princeton, NJ, 1970]. In this paper we prove that the Itô map, defined by $I(x)=y$, is a local $C^{k, \frac{\varepsilon}{1+\varepsilon}}$ map (in the sense of Fréchet) between $\Omega_{p}(V)$ and $\Omega_{p}(W)$, where $y$ is the solution to the differential equation

$$
d y_{t}=f\left(y_{t}\right) d x_{t}, \quad y_{0}=a .
$$

This result strengthens the continuity results and Lipschitz continuity results in [Lyons T., Differential equations driven by rough signals. I. An extension of an inequality of L.C. Young, Math. Res. Lett. 1 (4) (1994) 451-464; Lyons T., Qian Z., System Control and Rough Paths, Oxford Mathematical Monographs, Clarendon Press, Oxford, 2002] particularly to the non-integer case. It allows us to construct the fractional like Brownian motion and infinite dimensional Brownian motions on the space of paths with finite $p$-variation. As a corollary in the particular case where $p=1$, we obtain that the development from the space of finite 1 -variation paths on $\mathbb{R}^{d}$ to the space of finite 1 -variation paths on a $d$-dimensional compact Riemannian manifold is a smooth bijection.

(c) 2006 Elsevier Masson SAS

RÉSUMÉ. - Soient $p \in[1,2)$, et $\alpha$ tel que $\alpha \in(p-1,1-\varepsilon)$. Soient $V$ et $W$ deux espaces euclidiens. On désigne par $\Omega_{p}(V)$ l'espace des chemins continus à valeurs dans $V$ et de $p$-variation finie. Soit $f: W \rightarrow \operatorname{Hom}(V, W)$ une application de classe $\operatorname{Lip}(k+\alpha+\varepsilon)$ au sens de E.M. Stein, avec $k \in \mathbb{N}$ et $k \geqslant 1$. Dans cet article, nous montrons que l'application de Itô $I: \Omega_{p}(V) \rightarrow \Omega_{p}(W)$, définie par $I(x)=y$, où $y$ est la solution de l'équation différentielle suivante :

$$
d y_{t}=f\left(y_{t}\right) d x_{t}, \quad y_{0}=a,
$$

est localement de classe $C^{k, \frac{\varepsilon}{1+\varepsilon}}$ au sens de Fréchet. Cela nous permet de construire des processus de type mouvement brownien fractionnaire ainsi que des mouvements browniens de dimension infinie sur l'espace des chemins de $p$-variation finie. Comme corollaire, nous obtenons, dans le cas particulier où $p=1$, que l'application de développement de l'espace des chemins de 1-variation finie sur $\mathbb{R}^{d}$ dans l'espace des chemins de 1 -variation finie sur une variété riemannienne compacte $d$-dimensionnelle est une bijection régulière.

(c) 2006 Elsevier Masson SAS 


\section{Introduction}

Differential equations model the evolution of systems; they can be classified in a number of ways, and fall loosely into two basic classes:

(1) The deterministic or autonomous ones: these are specified in terms of a locally Lipschitz vector field $f$ which defines a differential equation

$$
\frac{d y_{t}}{d t}=f\left(y_{t}\right), \quad y_{0}=a
$$

(2) The controlled or non-autonomous ones: the essential feature that distinguishes these from the former is that one vector field defined the autonomous evolution, while the nonautonomous evolution is tangential to one of a collection of vector fields according to the choice of the controller, chance etc. Choosing a local basis, one might express these non-autonomous equations in the form

$$
\frac{d y_{t}}{d t}=\sum_{i} f^{i}\left(y_{t}\right) \frac{d x_{t}^{i}}{d t}, \quad y_{0}=a .
$$

The former setting embraces the classical theory of dynamical systems where today most interest revolves around long term behaviour, attractors, etc. In the latter setting there is a much richer local theory, with classical concrete examples giving considerable insight. The Cartan development of a smooth path from the tangent space or Lie algebra into the frame bundle or Lie group using the canonical vector fields provides an excellent example. See [2-4].

Note that Eq. (1.1) has an invariance: time re-parameterisation of $x$ and $y$ produces a new solution pair. Moreover one can simplify the expression by assuming vector notation and it is now commonplace to write

$$
d y_{t}=f\left(y_{t}\right) d x_{t}, \quad y_{0}=a
$$

and refer to the contact transformation taking the control $x$ to the response of the system $y$ as the Itô functional $I_{f}[11]$.

The mathematics of this second class of objects is rich and spans from Hopf algebras to technical aspects of analysis. One area of current research aims to understand the 'microlocal' structure and extend classical analysis to allow such equations to make sense when $x$ is not smooth. It is now understood that the Itô functional is defined for any rough ${ }^{1}$ path providing the vector fields $f$ are correspondingly smooth.

Interesting evolving systems are not necessarily finite dimensional. For example, there are many examples of real world dynamics where the state of the system is represented by a path or "a thread" that evolves or changes shape. Sometimes the evolution is parabolic in nature and can be dealt with via the techniques of PDE, where the path is regarded as a function and the "vector field" is the Laplace operator. But there are examples of a more hyperbolic nature where it seems that it is better to use modern mathematical methods to understand the vector fields and then use classical techniques to get solutions.

This paper is in the second direction. The aim of this paper is to develop a machinery allowing one to smoothly regard Itô functionals as the vector fields on path spaces. Our goal is to get sufficient control on the vector fields to allow one to apply the rough path theory to differential equations on path spaces.

\footnotetext{
${ }^{1}$ A technical term.

$4^{\mathrm{e}}$ SÉRIE - TOME $39-2006-\mathrm{N}^{\circ} 4$
} 
The Itô functional $x \rightarrow y$ produces one path from another; suppose that $y$ takes its values in a space $M$ and the control $x$ takes its values in a Banach space $E$ and that $F: M \rightarrow E$ is a smooth map, then $F(y)$ is a path in $E$. The path $F(y)$ can be regarded formally as a vector field on the space of paths in $E$ and one has a differential equation on path space

$$
\frac{d x}{d t}=F\left(I_{f}(x)\right)
$$

When correctly interpreted, Driver flows [7], as such equations are often called, have proved very natural and useful in probability theory, the classical Jacobi fields [21] and the geodesic equations on path and loop spaces [13-15] provide other examples with a similar flavour.

The analysis of such equations is not so straightforward. The basic Itô functional makes no sense for general continuous paths $x$. Even for bounded variation paths it is not so easy to establish the existence of the flow (which corresponds to a nonlinear hyperbolic PDE). Spaces of rough paths seem to provide the correct domain for these vector fields as $I_{f}(x)$ exists; it is proved in [20] that an evolution or flow does exist even for rough initial conditions. The solution may explode, but exists at least for a finite period of time; the existence of a flow makes it clear that the functional $F\left(I_{f}(x)\right)$ really is a vector field, and is not just a formal object; we can differentiate functions on rough path space in these directions. However, not much else is known, and for example the Lie Brackets of these fields, as well as the smoothness of these vector fields have remained obscure. This is a pity, as smoothness results might allow the direct construction of higher order differential operators, diffusions etc. on these spaces of paths.

The finite dimensional approach to solving a differential equation would have been to check that $I_{f}$ was Lipschitz, and then use the Picard theorem to get a solution. There is an understanding that this does not work in infinite dimensions because the interesting examples do not have this sort of smoothness; however we show carefully here that this is not correct. We prove that, as the vector fields $f$ get smoother, and for $1 \leqslant p<2$, the Itô map is appropriately differentiable in the Lipschitz sense. In this way we get very good control over the errors in system response as one varies a control, and in addition we are able to solve differential equations. Taking matters further we can even use the rough path theory again to solve stochastic differential equations on these spaces directly and so construct interesting and non-trivial flows and diffusions on path space.

These methods can be developed to discuss the Itô functional on the rough paths of any degree $p \geqslant 1$ and we expect to publish a paper on this second case. However, we believe that even in the bounded variation case $p=1$, our results are interesting as the differentiability of the control map as a function on paths of bounded variation already seems quite useful in engineering contexts. There is a phase transition of understanding between what happens for $1 \leqslant p<2$ and for rougher paths with many new concepts. The case $1 \leqslant p<2$ already exposes some of the essential ideas, and so it makes sense to separate out these ideas and to present this case out in detail.

\section{Part I - The linear case}

\subsection{Smoothness of Itô maps between path spaces}

Let $1 \leqslant p<2$. Let $V, W$ be two real separable Banach spaces. We denote by $\Omega_{p}(V)$ the collection of continuous paths from $[0,1]$ into $V$ with finite $p$-variation:

$$
\operatorname{Var}_{p}(x):=\sup _{\mathcal{D}} \sum_{t_{l} \in \mathcal{D}}\left\|x_{t_{l}}-x_{t_{l-1}}\right\|^{p}<\infty
$$


where $\mathcal{D}=\left\{0<t_{1}<\cdots<t_{n}=1\right\}, t_{0}=0$, and $\mathcal{D}$ ranges over all finite partitions of $[0,1]$. Then Wiener showed that $\Omega_{p}(V)$ is a Banach space with the norm

$$
\|x\|_{p}=\left[\operatorname{Var}_{p}(x)\right]^{1 / p}+\sup _{u \in[0,1]}\left\|x_{u}\right\| .
$$

It is straightforward that (1.2) has a solution in the classical sense whenever $x$ has bounded variation. The paths of bounded variation are dense in $\Omega_{p}(V)$ for every $p^{\prime}>p$ (see footnote ${ }^{2}$ ). By $[17,18,22]$, this mapping $x \rightarrow y$ extends uniquely to a Lipschitz continuous functional $I: \Omega_{p}(V) \rightarrow \Omega_{p}(W)$ provided that $f \in \operatorname{Lip}(\gamma)$ with $\gamma>p$. If $y=I(x)$, then $y$ solves the obvious integral equation and can be recovered as the limit in $p$-variation of the associated Picard iterations. Indeed, the uniform convergence of the Picard iteration process and the continuity of the iteration step ensure that $I: \Omega_{p}(V) \rightarrow \Omega_{p}(W)$ is a continuous and in fact a Lipschitz function in $x$. We refer to this map as the Itô map or Itô functional $I: \Omega_{p}(V) \rightarrow \Omega_{p}(W)$ and denote by $y$ the solution to the differential equation when driven by the rough path $x \in \Omega_{p}(V)$. In summary, we have the following

THEOREM $2.1[17,22]$. - Let $1 \leqslant p<2$ and $f: W \rightarrow H o m(V, W)$ be Lip $(\gamma)$ with $\gamma>p$. There exists a $R>0$ depending only on $\|f\|_{L i p(\gamma)}$ such that the differential equation

$$
d y_{t}=f\left(y_{t}\right) d x_{t}, \quad x_{0}=a
$$

has a unique solution $y \in \Omega_{p}(W)$ for any $x$ that has $p$-variation less than $R$. Any path $x \in \Omega_{p}([0,1], V)$ can be partitioned into finitely many pieces each of whose $p$-variation is less than $R$ and so a solution is available for all $x \in \Omega_{p}([0,1], V)$.

The case of a general $x$ can then be treated by a careful chaining together of these pieces of $x$. Moreover, the Itô map $I: \Omega_{p}([0,1], V) \rightarrow \Omega_{p}([0,1], W)$ is continuous and 1 -Lipschitz continuous with respect to the p-variation norms on bounded sets of paths.

Regarding $I: \Omega_{p}(V) \rightarrow \Omega_{p}(W)$ as a map between two Banach spaces, we can then ask whether there is a regularity condition on $f$ that forces the Itô map $I$ to be smooth in the sense of Fréchet.

Recall that for two real separable Banach spaces $E_{1}$ and $E_{2}$, a map $F: E_{1} \rightarrow E_{2}$ is called Fréchet differentiable if for all $x \in E_{1}$ there exists a bounded linear map denoted by $d F(x) \in$ $L\left(E_{1}, E_{2}\right)$ such that for some $\varepsilon>0$ and all $v \in B_{E_{1}}(0, \varepsilon)=\left\{v \in E_{1}:\|v\|_{E_{1}} \leqslant \varepsilon\right\}$ we have

$$
\|F(x+v)-F(x)-d F(x) v\|_{E_{2}}=o\left(\|v\|_{E_{1}}\right) .
$$

By induction, we can define the twice differentiability and $k$-times differentiability in the sense of Fréchet of a map $f: E_{1} \rightarrow E_{2}$. For this, see e.g. [6, Section 12, Chapter VIII]. As usual, we call $F: E_{1} \rightarrow E_{2}$ a smooth map in the sense of Fréchet if $F$ is $k$-times differentiable in the sense of Fréchet for all $k \in \mathbb{N}$.

Before stating the main results of this paper, let us recall that for all $0<\alpha<1$ the space $\operatorname{Lip}(\alpha)$ of Lipschitz continuous functions on $\mathbb{R}^{n}$ can be identified as $C^{0, \alpha}\left(\mathbb{R}^{n}, \mathbb{R}\right)$, and for any non-integer number $\alpha \in \mathbb{R}^{+}$, if we let $[\alpha]$ be the integer part of $\alpha$ and $\{\alpha\}=\alpha-[\alpha]$, then the space $\operatorname{Lip}(\alpha)$ can be identified as $C^{[\alpha],\{\alpha\}}\left(\mathbb{R}^{n}, \mathbb{R}\right)$. For this, we refer the reader to E.M. Stein [25, Section 4, Chapter V, pp. 141-150]. Here we follow Stein in calling a "Hölder continuous

\footnotetext{
${ }^{2}$ Precisely, the dyadic linear interpolations of a path $x$ have a $p$-variation at most $4^{p-1}$ times that of $x$ and converge to $x$ in $p^{\prime}$-variation for every $p^{\prime}>p$.

$4^{\mathrm{e}}$ SÉRIE - TOME $39-2006-\mathrm{N}^{\circ} 4$
} 
function" a Lipschitz function. We also mention that an essential part of the definition in Stein is that $f$ and all its derivatives of order less than $\alpha$ are bounded-in particular linear functions are not globally Lipschitz! This will also explain to some readers why the estimates in Theorem 2.1 do depend on $a$.

Now we are ready to state the main results of this paper as follows.

THEOREM 2.2. - Suppose that $V$ and $W$ are finite dimensional Banach spaces. Let $p \in[1,2)$, $\alpha, \varepsilon>0, \alpha \in(p-1,1-\varepsilon)$. Suppose that $f: W \rightarrow \operatorname{Hom}(V, W)$ is in Lip $(k+\alpha+\varepsilon)$ where $k \in \mathbb{N}$ and $k \geqslant 1$. Then the Itô map $I: \Omega_{p}(V) \rightarrow \Omega_{p}(W)$ is locally $k$-times Fréchet-differentiable. More precisely, if we work in the Euclidean norms on $V, W$ there exists a constant $K$ depending only on $p, \alpha, k, \varepsilon$ and $\|f\|_{k+\alpha+\varepsilon}$ such that for all $x \in \Omega_{p}$ with $\|x\|_{p} \leqslant K$, there exists a bounded $k$-linear operator, denoted by $I^{(k)}(x) \in L_{n}\left(\Omega_{p}(V), \Omega_{p}(W)\right)$, such that for $v_{1}, \ldots, v_{k} \in \Omega_{p}(V)$ with $\left\|v_{1}\right\|_{p}, \ldots,\left\|v_{k}\right\|_{p} \leqslant K$, and for $i=1, \ldots, k$, we have the Gâteaux derivatives

$$
I^{(i)}(x)\left(v_{1}, \ldots, v_{i}\right):=\left.\left\{\frac{\partial^{i}}{\partial \varepsilon_{1} \cdots \partial \varepsilon_{i}} I\left(x+\sum_{j=1}^{i} \varepsilon_{j} v_{j}\right)\right\}\right|_{\varepsilon_{1}=\cdots=\varepsilon_{i}=0}
$$

exist in $\Omega_{p}(W)$ with respect to the p-variation norm, and I is Fréchet differentiable

$$
\left\|I(x+v)-I(x)-\sum_{i=1}^{k} \frac{I^{(i)}(x) v^{\otimes i}}{i !}\right\|_{p} \leqslant C\|v\|_{p}^{k+\frac{\varepsilon}{1+\varepsilon}} .
$$

Here $C$ is a constant depending only on $p, \alpha, \varepsilon,\|f\|_{k+\alpha+\varepsilon},\|x\|_{p} \leqslant K$ and $\|v\|_{p} \leqslant K$.

The proof of Theorem 2.2 is based on the Fréchet differentiability of the Picard iteration $I_{n}: \Omega_{p}(V) \rightarrow \Omega_{p}(W), y_{n}=I_{n}(x)$, of the differential equation (1.2) given by

$$
y_{n+1}(t)=a+\int_{0}^{t} f\left(y_{n}(s)\right) d x(s)
$$

We shall prove that $I_{n}: \Omega_{p}(V) \rightarrow \Omega_{p}(W)$ is locally $k$-times differentiable in the sense of Fréchet and satisfies the estimate

$$
\left\|I_{n}(x+v)-I_{n}(x)-\sum_{i=1}^{k} \frac{I^{(i)}(x) v^{\otimes i}}{i !}\right\|_{p} \leqslant C\|v\|_{p}^{k+\frac{\varepsilon}{1+\varepsilon}}
$$

with a uniform constant $C$ that does not depend on $n$. To this end, we shall first prove that the Young integral

$$
(x, y) \rightarrow \int_{0} f(y(s)) d x(s)
$$

is $k$-times Fréchet differentiable from $\Omega_{p}(V) \times \Omega_{p}(W)$ into $\Omega_{p}(W)$ under the assumption $f \in \operatorname{Lip}(k+\alpha+\varepsilon)$. By the chain rule and induction, we need only to prove that the map $\Phi_{f}: \Omega_{p}(V) \rightarrow \Omega_{\frac{p}{\alpha}}(W)$ defined by

$$
\Phi_{f}(x)=f(x)
$$

ANNALES SCIENTIFIQUES DE L'ÉCOLE NORMALE SUPÉRIEURE 
is smooth in the sense of Fréchet. The complete proof of Theorem 2.2 will be given in Sections 2.3 and 2.4. Moreover, we can further prove that if $f \in C^{k+1, \alpha}$ with $p-1<\alpha<1$, then

$$
\left\|I(x+v)-I(x)-\sum_{i=1}^{k} \frac{I^{(i)}(x) v^{\otimes i}}{i !}\right\|_{p} \leqslant C\|v\|_{p}^{k+1} .
$$

Here $C$ is a constant depending only on $p, \alpha, \varepsilon,\|f\|_{k+1, \alpha},\|x\|_{p} \leqslant K$ and $\|v\|_{p} \leqslant K$.

We emphasise that our techniques establish a rigorous machinery for considering many concrete problems on path space. The space $\Omega_{1}(M)$ comprises bounded variation paths on a compact Riemannian manifold. Many interesting computational problems arising in variational calculus on Riemannian manifolds aim to approximate (or identify) the global minimum or global maximum of a smooth functional $F: \Omega_{1}(M) \rightarrow \mathbb{R}$. The smoothness of the Itô map $I: \Omega_{p}\left(\mathbb{R}^{d}\right) \rightarrow \Omega_{p}(M)$ allows us to study the optimisation problems on path spaces by transferring the optimisation problem of a smooth $F: \Omega_{p}(M) \rightarrow \mathbb{R}$ to a corresponding problem for the map $\widetilde{F}=F \circ I: x \rightarrow \widetilde{F}(x)=F(I(x))$.

In the last section we point out that we have developed an infinite dimensional framework where the classical finite dimensional numerical tools (from steepest descent to simulated annealing) can be used. Of course, each specific example has essential features that would have to be addressed before real progress could be claimed (e.g. is the minimum attained). We cannot begin to answer these within this paper and would be delighted if we stimulate others to take up the challenges.

\subsection{Smoothness of the Young integral}

To prove the Fréchet smoothness of the Young integral $(x, y) \rightarrow \int_{0}^{\cdot} f(y) d x$ under the assumption $f \in \operatorname{Lip}(1+\alpha+\varepsilon)$, where $\alpha \in(0,1), \varepsilon>0$, we first prove that the map $\Phi_{f}: \Omega_{p}(V) \rightarrow \Omega_{\frac{p}{\alpha}}(W)$ defined by

$$
\Phi_{f}(x)=f(x)
$$

is smooth in the sense of Fréchet. It is interesting to note that our proof is essentially finite dimensional and exploits basic harmonic analysis, for example, Lemmas 2.9, 2.11 and Lemma 2.12 take the advantage of the Poisson integral formula and the characterisation of Lipschitz functions in terms of the partial derivatives of their harmonic extension.

Below we collect some elementary facts and results on the $p$-variation norm and the Young integral. Some proofs are easy and will be omitted. The reader can consult $[9,17,18,22]$ for the definition of the Young integral. Theorem 2.17 is our main result in this section.

Proposition 2.3. - Let $x \in \Omega_{p}(V), y \in \Omega_{q}(W), p \geqslant q$. Then $x y \in \Omega_{p}(V \otimes W)$. Moreover, there exists a constant $C=C_{p}>0$ such that

$$
\|x \otimes y\|_{[s, t], p} \leqslant C\|x\|_{\infty}\|y\|_{q}+C\|y\|_{\infty}\|x\|_{p} .
$$

Here $V \otimes W$ is the tensor product of $V$ and $W,\|\cdot\|_{V \otimes W}$ is any compatible norm on $V \otimes W$.

COROLLARY 2.4. - For any $1<p<2$, there exists a constant $C_{p}$ such that

$$
\|x y\|_{p} \leqslant C_{p}\|x\|_{p}\|y\|_{p} .
$$

For any $s, t \in[0, \infty), s<t$, we denote by $\|x\|_{[s, t], p}$ the $p$-variation norm of $x:[s, t] \rightarrow V$. We now recall the so-called Young-Love inequality. For a proof, see $[9,17]$.

$4^{\text {e }}$ SÉRIE - TOME $39-2006-\mathrm{N}^{\circ} 4$ 
Proposition 2.5. - Let $x \in \Omega_{p}(V), y \in \Omega_{q}(W)$. If $\frac{1}{p}+\frac{1}{q}>1$, then the Young integral $\int_{0}^{\cdot} y_{s} \otimes d x_{s}$ exists and is a finite $p$-variation path. Moreover,

$$
\left\|\int_{0} y \otimes d x\right\|_{[s, t], p} \leqslant\left(\xi(1 / p+1 / q)\|y\|_{[s, t], q}+\|y\|_{\infty}\right)\|x\|_{[s, t], p}
$$

Proposition 2.6. - Let $x \in \Omega_{p}(V), y \in \Omega_{q}(W)$. Then $F:(x, y) \rightarrow \int_{0}^{\cdot} y \otimes d x$ is a $C^{\infty}$. smooth map from $\Omega_{p}(V) \times \Omega_{q}(W) \rightarrow \Omega_{p}(V \otimes W)$. Moreover, the first order derivative of $F$ at $(x, y)$ is given by

$$
(v, w) \rightarrow \int_{0} w d x+\int_{0} y d v
$$

Proposition 2.7. - Let $x \in \Omega_{p}(V), \alpha \in(0,1)$, and $f \in C^{0, \alpha}(V, W)$. Then $y=f(x) \in$ $\Omega_{q}(W)$, for $q \geqslant \frac{p}{\alpha}$. Moreover

$$
\|f(x)\|_{[s, t], \frac{p}{\alpha}} \leqslant\|f\|_{C^{0, \alpha},\|x\|_{[s, t], p}^{\alpha}}^{\alpha}
$$

Proof. - Let $\|f\|_{C^{0, \alpha}}$ be the Hölder norm of $f$. Then

$$
\left\|f\left(x_{s_{i}}\right)-f\left(x_{s_{i-1}}\right)\right\|_{W} \leqslant\|f\|_{C^{0, \alpha}}\left\|x_{s_{i}}-x_{s_{i-1}}\right\|_{V}^{\alpha} .
$$

By a standard argument, it is easy to see that $f(x)$ is a finite $q$-variation path for all $q \geqslant \frac{p}{\alpha}$ and so (2.5) holds.

Combining Proposition 2.7 and the Young-Love inequality, we have the following

COROLlaRY 2.8. - Let $x \in \Omega_{p}\left(V_{1}\right), \quad y \in \Omega_{q}\left(V_{2}\right), \quad \alpha \in(0,1)$, and for all $f \in C^{0, \alpha}\left(V_{2}, L\left(V_{1}, W\right)\right)$. If $\frac{1}{p}+\frac{\alpha}{q}>1$, then $z=\int_{0}^{\cdot} f(y) d x \in \Omega_{p}(W)$. Moreover,

$$
\left\|\int_{0} f(y) d x\right\|_{[s, t], p} \leqslant\left[\xi(1 / p+\alpha / q)\|f\|_{C^{0, \alpha}}\|y\|_{q}^{\alpha}+\|f\|_{\infty}\right]\|x\|_{[s, t], p} .
$$

We need two technical lemmas. For a function $f \in \operatorname{Lip}(\alpha), \alpha>0$ and defined on $\mathbb{R}^{m}$ we introduce the Poisson integral of $f$ by

$$
f_{t}(x)=\frac{\Gamma\left(\frac{m+1}{2}\right)}{\pi^{\frac{m+1}{2}}} \int \frac{f(x-y) t}{\left(t^{2}+|y|^{2}\right)^{\frac{m+1}{2}}} d y, \quad t>0 .
$$

LEMMA 2.9. - For any $0<\beta<\alpha$, we have

$$
\left\|f_{t}\right\|_{\alpha} \leqslant C_{\alpha, \beta} \mid f \|_{\beta} t^{\beta-\alpha}, \quad \forall t>0 .
$$

Proof. - By [25, Section 4.3, p. 145], we see that $f \in \operatorname{Lip}(\beta)$ with the norm $\|f\|_{\beta}$ if and only if for $k=[\beta]+1$,

$$
\left\|\frac{\partial^{k}}{\partial y^{k}} f_{y}\right\|_{\infty} \leqslant\|f\|_{\beta} y^{\beta-k}, \quad \forall y>0 .
$$

ANNALES SCIENTIFIQUES DE L'ÉCOLE NORMALE SUPÉRIEURE 
Hence for all $t>0$ and $y>0$, we have

$$
\left\|\frac{\partial^{k}}{\partial y^{k}} f_{y+t}\right\|_{\infty} \leqslant\|f\|_{\beta}(y+t)^{-k+\beta}
$$

and therefore

$$
\left\|\frac{\partial^{k}}{\partial y^{k}} f_{y+t}\right\|_{\infty} \leqslant\|f\|_{\beta} \sup _{y>0}\left[\frac{y^{k-\alpha}}{(y+t)^{k-\beta}}\right] y^{-k+\alpha} .
$$

Now it is easy to see that $\sup _{y>0}\left[\frac{y^{k-\alpha}}{(y+t)^{k-\beta}}\right]$ is attained at $y=\frac{k-\alpha}{\alpha-\beta} t$. Thus

$$
\sup _{y>0}\left[\frac{y^{k-\alpha}}{(y+t)^{k-\beta}}\right]=C_{\alpha, \beta, k} t^{\beta-\alpha} .
$$

Hence

$$
\left\|\frac{\partial^{k}}{\partial y^{k}} f_{y+t}\right\|_{\infty} \leqslant C_{\alpha, \beta, t} t^{\beta-\alpha}\|f\|_{\beta} y^{-k+\alpha}, \quad \forall y>0,
$$

which is equivalent to

$$
\left\|f_{t}\right\|_{\alpha}<C_{\alpha, \beta}\|f\|_{\beta} t^{\beta-\alpha} .
$$

Proposition 2.10. - Let $\beta>0, \varepsilon>0, \beta+\varepsilon<1$. Then there exists a constant $C=C_{\beta, \varepsilon}$ such that

$$
y^{\beta+\varepsilon-1}-(y+h)^{\beta+\varepsilon-1} \leqslant C y^{\beta-1} h^{\varepsilon}, \quad \forall y>0, h>0 .
$$

Proof. - Let $h=z y$. Then the desired inequality is equivalent to

$$
1-(1+z)^{\beta+\varepsilon-1} \leqslant C z^{\varepsilon}, \quad \forall z>0 .
$$

Let

$$
F_{C}(z)=C z^{\varepsilon}+(1+z)^{\beta+\varepsilon-1}, \quad z \geqslant 0
$$

Then

$$
F_{C}(0)=0
$$

We need only to prove that for any $\beta \in(0,1), \varepsilon \in(0,1-\beta)$ there exists a constant $C=C_{\beta, \varepsilon}$ such that

$$
F_{C}^{\prime}(z)>0, \quad \forall z>0
$$

Note that

$$
F_{C}^{\prime}(z)=\varepsilon C z^{\varepsilon-1}+(\beta+\varepsilon-1)(1+z)^{\beta+\varepsilon-2} .
$$

Moreover, the inequality $F_{C}^{\prime}(z)>0$ holding for all $z>0$ is equivalent to

$$
\gamma:=\inf _{z>0} \log \frac{z^{\varepsilon-1}}{(1+z)^{\beta+\varepsilon-2}}>-\infty .
$$

Indeed, if $\gamma>-\infty$, then for all

$$
C \geqslant \frac{1-\beta-\varepsilon}{\varepsilon e^{\gamma}}
$$

$4^{\mathrm{e}}$ SÉRIE - TOME $39-2006-\mathrm{N}^{\circ} 4$ 
we have $F_{C}^{\prime}(z)>0$ for all $z>0$.

Below we prove $\gamma>-\infty$. Set

$$
G_{\beta, \varepsilon}(z)=\log \frac{z^{\varepsilon-1}}{(1+z)^{\beta+\varepsilon-2}}, \quad z>0 .
$$

Then

$$
G_{\beta, \varepsilon}=(\varepsilon-1) \log z-(\beta+\varepsilon-2) \log (z+1)
$$

and

$$
G_{\beta, \varepsilon}(z)=G_{\varepsilon, \beta}\left(z^{-1}\right), \quad z>0 .
$$

Moreover,

$$
\lim _{z \rightarrow 0+} G_{\beta, \varepsilon}(z)=\lim _{z \rightarrow 0+} G_{\varepsilon, \beta}(z)=+\infty .
$$

Combining this with the continuity of $G_{\beta, \varepsilon}(z)$ with respect to $z \in(0,+\infty)$, we get

$$
\gamma:=\inf _{z>0} G_{\beta, \varepsilon}(z)>-\infty
$$

LEMMA 2.11. - For any $h>0$, we have

$$
\left\|f_{h}-f\right\|_{\alpha} \leqslant C\|f\|_{\alpha+\varepsilon} h^{\varepsilon} .
$$

Proof. - Using the same argument as used in the proof of Lemma 2.9, for sufficiently small $\varepsilon>0$ we have

$$
\begin{aligned}
\left\|\frac{\partial^{k}}{\partial y^{k}}\left(f_{y+h}-f_{y}\right)\right\| & =\left\|\frac{\partial^{k}}{\partial y^{k}} \int_{0}^{1} \frac{\partial}{\partial y} f_{y+\theta h} h d \theta\right\| \\
& \leqslant \int_{0}^{1}\left\|\frac{\partial^{k+1}}{\partial y^{k+1}} f_{y+\theta h}\right\| h d \theta \\
& \leqslant\|f\|_{\alpha+\varepsilon} \int_{0}^{1}(y+\theta h)^{-(k+1)+\alpha+\varepsilon} h d \theta \\
& =(k-\alpha-\varepsilon)^{-1}\|f\|_{\alpha+\varepsilon}\left[y^{-k+\alpha+\varepsilon}-(y+h)^{-k+\alpha+\varepsilon}\right] .
\end{aligned}
$$

By Proposition 2.10, for all $y>0$ and $h>0$, we have

$$
y^{-k+\alpha+\varepsilon}-(y+h)^{-k+\alpha+\varepsilon} \leqslant C_{k, \alpha, \beta} y^{-k+\alpha} h^{\varepsilon} .
$$

Hence

$$
\left\|\frac{\partial^{k}}{\partial y^{k}}\left(f_{y+h}-f_{y}\right)\right\| \leqslant C_{k, \alpha, \varepsilon}\|f\|_{\alpha+\varepsilon} h^{\varepsilon} y^{-k+\alpha} .
$$

which is equivalent to

$$
\left\|f_{h}-f\right\|_{\alpha} \leqslant C\|f\|_{\alpha+\varepsilon} h^{\varepsilon} .
$$

ANNALES SCIENTIFIQUES DE L'ÉCOLE NORMALE SUPÉRIEURE 
LEMmA 2.12. - Let $f \in \operatorname{Lip}(1+\alpha), 0<\alpha<1$. Then for any $K>0$ and for any $x, y \in \Omega_{p}(V)$ with $\|x\|_{p} \leqslant K$ and $\|y\|_{p} \leqslant K$, we have

$$
\|f(x)-f(y)\|_{\frac{p}{\alpha}} \leqslant C_{\alpha, K}\|f\|_{1+\alpha}\|x-y\|_{p} .
$$

Proof. - By the mean value theorem we have

$$
f(x)-f(y)=\int_{0}^{1} f^{\prime}(y+\theta(x-y))(x-y) d \theta .
$$

Hence

$$
\begin{aligned}
\|f(x)-f(y)\|_{\frac{p}{\alpha}} & \leqslant \max _{\theta \in[0,1]}\left\|f^{\prime}(y+\theta(x-y))(x-y)\right\|_{\frac{p}{\alpha}} \\
& \leqslant \max _{\theta \in[0,1]}\left\|f^{\prime}(y+\theta(x-y))\right\|_{\frac{p}{\alpha}}\|x-y\|_{\frac{p}{\alpha}} \\
& \leqslant \max _{\theta \in[0,1]}\left[\left\|f^{\prime}\right\|_{\alpha}\|y+\theta(x-y)\|_{p}^{\alpha}\right]\|x-y\|_{p} \\
& \leqslant\|f\|_{1+\alpha}\left[\|y\|_{p}^{\alpha}+\|x-y\|_{p}^{\alpha}\right]\|x-y\|_{p} .
\end{aligned}
$$

Thus

$$
\|f(x)-f(y)\|_{\frac{p}{\alpha}} \leqslant C\|f\|_{1+\alpha}\|x-y\|_{p}
$$

with

$$
C_{\alpha, K}=3 K^{\alpha}
$$

THEOREM 2.13. - Let $f \in \operatorname{Lip}(\alpha+\varepsilon)$ and $\alpha \in(0,1-\varepsilon)$. Then $x \rightarrow f(x)$ is $\frac{\varepsilon}{1+\varepsilon}$-Lipschitz continuous from $\Omega_{p}$ into $\Omega_{\frac{p}{\alpha}}$. More precisely,

$$
\|f(x)-f(y)\|_{\frac{p}{\alpha}} \leqslant C_{p, \varepsilon, \alpha}\|f\|_{\alpha+\varepsilon}\|x-y\|_{p}^{\frac{\varepsilon}{1+\varepsilon}} .
$$

Proof. - Let $f_{h}$ be the Poisson integral of $f$ on $\mathbb{R}^{m} \times \mathbb{R}^{+}, h>0$. By Lemmas 2.9, 2.11 and Lemma 2.12, we have

$$
\begin{aligned}
\|f(x)-f(y)\|_{\frac{p}{\alpha}} & \leqslant\left\|f-f_{h}\right\|_{\alpha}\left(\|x\|_{p}^{\alpha}+\|y\|_{p}^{\alpha}\right)+\left\|f_{h}\right\|_{1+\alpha}\|x-y\|_{p} \\
& \leqslant C_{1} h^{\varepsilon}\|f\|_{\alpha+\varepsilon}+C_{2} \frac{\|f\|_{\alpha}}{h}\|x-y\|_{p} .
\end{aligned}
$$

Hence

$$
\begin{aligned}
\|f(x)-f(y)\|_{\frac{p}{\alpha}} & \leqslant \inf _{h>0}\left[C_{1} h^{\varepsilon}\|f\|_{\alpha+\varepsilon}+C_{2} \frac{\|f\|_{\alpha}}{h}\|x-y\|_{p}\right] \\
& \leqslant\|f\|_{\alpha+\varepsilon} \inf _{h>0}\left[C_{1} h^{\varepsilon}+\frac{C_{2}\|x-y\|_{p}}{h}\right] .
\end{aligned}
$$

It is easy to show that the infimum of the right-hand side is attained at

$$
h=C_{3}\|x-y\|_{p}^{\frac{1}{1+\varepsilon}} .
$$

$4^{\text {e }}$ SÉRIE - TOME $39-2006-\mathrm{N}^{\circ} 4$ 
Substituting this into the previous inequality, we get

$$
\|f(x)-f(y)\|_{\frac{p}{\alpha}} \leqslant C_{p, \varepsilon, \alpha}\|f\|_{\alpha+\varepsilon}\|x-y\|_{p}^{\frac{\varepsilon}{1+\varepsilon}}
$$

The following lemma holds even for infinite dimensional Banach spaces $V$ and is used in the proof of our main theorem. This representation of differences as integrals seems critical and appears in slight different forms in

Lemma 2.14. - Let $f \in \operatorname{Lip}(2+\alpha)$ with $0<\alpha<1$. Then for any $K>0$ and for any $x, y \in \Omega_{p}(V)$ with $\|x\|_{p} \leqslant K$ and $\|y\|_{p} \leqslant K$, there exists a constant $C_{K}$ such that

$$
\left\|f(x)-f(y)-f^{\prime}(x)(x-y)\right\|_{\frac{p}{\alpha}} \leqslant C_{K}\|f\|_{2+\alpha}\|x-y\|_{p}^{2} .
$$

Proof. - Let $R(x, y)=f(x)-f(y)-f^{\prime}(x)(x-y)$. By the mean value theorem,

$$
R_{f}(x, y)=\int_{0}^{1}(1-\theta) f^{\prime \prime}(y+\theta(x-y))(x-y, x-y) d \theta .
$$

Hence

$$
\begin{aligned}
\left\|R_{f}(x, y)\right\|_{\frac{p}{\alpha}} & \leqslant \max _{\theta \in[0,1]}\left\|f^{\prime \prime}(y+\theta(x-y))\right\|_{\frac{p}{\alpha}}\|x-y\|_{\frac{p}{\alpha}}^{2} \\
& \leqslant\left\|f^{\prime \prime}\right\|_{\alpha} \max _{\theta \in[0,1]}\|y+\theta(x-y)\|_{p}^{\alpha}\|x-y\|_{p}^{2} \\
& \leqslant\|f\|_{2+\alpha}\left[\|y\|_{p}^{\alpha}+\|x-y\|_{p}^{\alpha}\right]\|x-y\|_{p}^{2} .
\end{aligned}
$$

Thus for $C_{K}=3 K^{\alpha}$, we have

$$
\left\|R_{f}(x, y)\right\|_{\frac{p}{\alpha}} \leqslant C_{K}\|f\|_{2+\alpha}\|x-y\|_{p}^{2}
$$

The following result uses our estimates that depended on the Poisson extension and plays a crucial role in the proof of our main theorem.

THEOREM 2.15. - Let $f \in \operatorname{Lip}(1+\alpha+\varepsilon), \alpha \in(p-1,1-\varepsilon)$. Then $\Phi_{f}: x \rightarrow f(x)$ is smooth in the sense of Fréchet from $\Omega_{p}\left(\mathbb{R}^{m}\right)$ into $\Omega_{\frac{p}{\alpha}}\left(\mathbb{R}^{l}\right)$. Moreover, there exists a constant $C=C(p, \alpha, \varepsilon)$ such that

$$
\left.\left\|\Phi_{f}(y)-\Phi_{f}(x)-\Phi_{f}^{\prime}(x)(y-x)\right\|\right|_{\frac{p}{\alpha}} \leqslant C\|f\|_{1+\alpha+\varepsilon}\|x-y\|_{p}^{\frac{1+2 \varepsilon}{1+\varepsilon}} .
$$

Proof. - By definition, we need to prove

$$
\left\|f(y)-f(x)-f^{\prime}(x)(y-x)\right\|_{\frac{p}{\alpha}} \leqslant C\|f\|_{1+\alpha+\varepsilon}\|x-y\|_{p^{\frac{1+2 \varepsilon}{1+\varepsilon}}} .
$$

Let $f_{h}$ be the harmonic extension of $f$ on $\mathbb{R}^{m} \times \mathbb{R}^{+}$. Using Lemmas 2.12 and 2.14, we have

$$
\begin{aligned}
\left\|R_{f}(x, y)\right\|_{\frac{p}{\alpha}} \leqslant & \left\|R_{f-f_{h}}(x, y)\right\|_{\frac{p}{\alpha}}+\left\|R_{f_{h}}(x, y)\right\|^{\leqslant} \\
& \left\|f(x)-f_{h}(x)-\left(f(y)-f_{h}(y)\right)\right\|_{\frac{p}{\alpha}} \\
& +\left\|\left(f-f_{h}\right)^{\prime}(x)(x-y)\right\|_{\frac{p}{\alpha}}+\left\|R_{f_{h}}(x, y)\right\|_{\frac{p}{\alpha}}
\end{aligned}
$$

ANNALES SCIENTIFIQUES DE L'ÉCOLE NORMALE SUPÉRIEURE 


$$
\begin{aligned}
\leqslant & C_{1}\left\|f-f_{h}\right\|_{1+\alpha}\|x-y\|_{p}+\left\|\left(f-f_{h}\right)^{\prime}(x)\right\|_{\frac{p}{\alpha}}\|x-y\|_{\frac{p}{\alpha}} \\
& +C_{2}\left\|f_{h}\right\|_{2+\alpha}\|x-y\|_{p}^{2} \\
\leqslant & C_{2}\left\|f_{h}\right\|_{2+\alpha}\|x-y\|_{p}^{2}+C_{1}\left\|f-f_{h}\right\|_{1+\alpha}\|x-y\|_{p} \\
& +\left\|\left(f-f_{h}\right)^{\prime}\right\|_{\alpha}\|x\|_{p}^{\alpha}\|x-y\|_{p} \\
\leqslant & C_{2}\left\|f_{h}\right\|_{2+\alpha}\|x-y\|_{p}^{2} \\
& +\left[C_{1}\left\|f-f_{h}\right\|_{1+\alpha}+\left\|\left(f-f_{h}\right)^{\prime}\right\|_{\alpha}\|x\|_{p}^{\alpha}\right]\|x-y\|_{p} \\
\leqslant & C_{2}\left\|f_{h}\right\|_{2+\alpha}\|x-y\|_{p}^{2}+C_{3}\left\|f-f_{h}\right\|_{1+\alpha}\|x-y\|_{p} .
\end{aligned}
$$

Now by Lemmas 2.9 and 2.11, we have

$$
\begin{aligned}
& \left\|f_{h}\right\|_{2+\alpha} \leqslant C_{4} \frac{\|f\|_{1+\alpha}}{h}, \\
& \left\|f-f_{h}\right\|_{1+\alpha} \leqslant C_{5}\|f\|_{1+\alpha+\varepsilon} h^{\varepsilon} .
\end{aligned}
$$

Hence for all $h>0$,

$$
\begin{aligned}
\left\|R_{f}(x, y)\right\|_{\frac{p}{\alpha}} & \leqslant C_{6}\|x-y\|_{p}^{2}\|f\|_{1+\alpha} h^{-1}+C_{7}\|x-y\|_{p}\|f\|_{1+\alpha+\varepsilon} h^{\varepsilon} \\
& \leqslant\|f\|_{1+\alpha+\varepsilon}\|x-y\|_{p}\left[C_{6}\|x-y\|_{p} h^{-1}+C_{7} h^{\varepsilon}\right] .
\end{aligned}
$$

Taking the infimum over $h$ we get

$$
\left\|R_{f}(x, y)\right\|_{\frac{p}{\alpha}} \leqslant C_{8}\|f\|_{1+\alpha+\varepsilon}\|x-y\|_{p}^{\frac{1+2 \varepsilon}{1+\varepsilon}} .
$$

By repeating the argument as set out above, we can prove a general result as follows

THEOREM 2.16. - Let $f \in \operatorname{Lip}(k+\alpha+\varepsilon), \alpha \in(p-1,1-\varepsilon)$. Then there exists a constant $C=C(k, \alpha, \varepsilon)$ such that

$$
\left\|\Phi_{f}(y)-f(x)-\sum_{i=1}^{k} \frac{\Phi_{f^{(i)}}(x)(x-y)^{\otimes i}}{i !}\right\|_{\frac{p}{\alpha}} \leqslant C\|f\|_{k+\alpha+\varepsilon}\|x-y\|_{p}^{k+\frac{\varepsilon}{1+\varepsilon}},
$$

where $\Phi_{f^{(i)}}(x):=f^{(i)}(x), i=0, \ldots, k, x \in \Omega_{p}\left(\mathbb{R}^{n}\right)$.

Now we are ready to prove the main result of this section. Let $V_{1}$ and $V_{2}$ be two finite dimensional Banach spaces.

THEOREM 2.17. - Let $x \in \Omega_{p}\left(V_{1}\right), y \in \Omega_{q}\left(V_{2}\right), f \in \operatorname{Lip}\left(1+\alpha+\varepsilon, V_{2}, L\left(V_{1}, W\right)\right)$. If $\frac{1}{p}+\frac{\alpha}{q}>1$, then $\Phi:(x, y) \rightarrow \int_{0}^{x} f(y) d x$ is differentiable in the sense of Fréchet from $\Omega_{p}\left(V_{1}\right) \times \Omega_{q}\left(V_{2}\right)$ into $\Omega_{p}(W)$. Moreover, the Gâteaux derivative of $\Phi$ at $(x, y)$ is given by

$$
\Phi^{\prime}(x, y)(v, w)=\int_{0} f^{\prime}\left(y_{s}\right) w_{s} d x_{s}+\int_{0}^{j} f\left(y_{s}\right) d v_{s} .
$$

\section{Moreover,}

$$
\left\|\int_{0} f(y) d v\right\|_{[s, t], p} \leqslant\left[\xi(1 / p+\alpha / q)\|f\|_{C^{0, \alpha}}\|y\|_{[s, t], q}^{\alpha}+\|f\|_{\infty}\right]\|v\|_{[s, t], p},
$$

$4^{\text {e }}$ SÉRIE - TOME $39-2006-\mathrm{N}^{\circ} 4$ 


$$
\begin{aligned}
\left\|\int_{0} f^{\prime}(y) w d x\right\|_{[s, t], p} \leqslant & {\left[\xi(1 / p+\alpha / q)\left\|f^{\prime}\right\|_{C^{0, \alpha}}\|y\|_{q}^{\alpha}+\left\|f^{\prime}\right\|_{\infty}\right] } \\
& \times\left[\xi(1 / p+1 / q)\|w\|_{[s, t], q}+\|w\|_{\infty}\right]\|x\|_{[s, t], p} .
\end{aligned}
$$

Proof. - Let $F$ be as in Proposition 2.6, i.e., $F(x, y)=\int_{0}^{\cdot} y d x$. Then

$$
\Phi(x, y)=F(x, f(y))
$$

Combining Theorem 2.15 with Proposition 2.6 and using the chain rule, we can conclude that the Young integral $\Phi$ is Fréchet differentiable from $\Omega_{p}\left(V_{1}\right) \times \Omega_{q}\left(V_{2}\right)$ into $\Omega_{p}(W)$. Moreover, for $(x, y),(v, w) \in \Omega_{p}\left(V_{1}\right) \times \Omega_{p}\left(V_{2}\right)$, we have

$$
\Phi^{\prime}(x, y)(v, w)=F^{\prime}(x, f(y)) \circ\left(I d, f^{\prime}(y)\right)(v, w) .
$$

Hence

$$
\begin{aligned}
\Phi^{\prime}(x, y)(v, w) & =F^{\prime}(x, f(y))\left(v, f^{\prime}(y) w\right) \\
& =F\left(x, f^{\prime}(y) w\right)+F(v, f(y)) \\
& =\int_{0} f^{\prime}\left(y_{s}\right) w_{s} d x_{s}+\int_{0} f\left(y_{s}\right) d v_{s} .
\end{aligned}
$$

The estimate on $\left\|\int_{0}^{\cdot} f(y) d v\right\|_{[s, t], p}$ follows from Corollary 2.8. By the Young-Love inequality, we have

$$
\begin{aligned}
\left\|\int_{0} f^{\prime}(y) w d x\right\|_{[s, t], p} \leqslant & {\left[\xi(1 / p+\alpha / q)\left\|f^{\prime}\right\|_{C^{0, \alpha}}\|y\|_{q}+\left\|f^{\prime}\right\|_{\infty}\right]\left\|\int_{0} w d x\right\|_{[s, t], p} } \\
\leqslant & {\left[\xi(1 / p+\alpha / q)\left\|f^{\prime}\right\|_{C^{0, \alpha}}\|y\|_{q}+\left\|f^{\prime}\right\|_{\infty}\right] } \\
& \times\left[\xi(1 / p+1 / q)\|w\|_{q}+\|w\|_{\infty}\right]\|x\|_{[s, t], p}
\end{aligned}
$$

\subsection{Uniform convergence of the derivatives of the Picard iterates}

Recall that our goal is to prove the Fréchet differentiability of the Itô map $I: \Omega_{p}(V) \rightarrow$ $\Omega_{p}(W)$ with a quantitative control. The Itô map is the limit of Picard iterates in the same way that the exponential function $e^{x}$ is the limit of a sequence of polynomials $\sum_{k=0}^{n} \frac{x^{k}}{k !}$. Every undergraduate knows that, as each of these polynomials is differentiable and their derivatives converge uniformly (to $e^{x}$ ), the exponential function $e^{x}$ is differentiable (with derivative $e^{x}$ ). In Section 2.2 we proved that the Picard iterates are Fréchet differentiable. If we can prove their derivatives converge uniformly on bounded sets we will have our main result on the differentiability of the Itô map.

Let $I_{n}: \Omega_{p}(V) \rightarrow \Omega_{p}(W)$ be the $n$-th Picard iterate, given by $I_{0}(x)=y_{0}=a, I_{n}(x)=y_{n}$, where $y_{n}$ is defined recursively:

$$
y_{n+1}(t)=a+\int_{0}^{t} f\left(y_{n}(s)\right) d x(s) .
$$


ThEOREM 2.18. - Let $f \in \operatorname{Lip}(1+\alpha+\varepsilon)$ with $\alpha \in(p-1,1-\varepsilon)$. Then $I_{n}: \Omega_{p} \rightarrow \Omega_{p}$ is Fréchet differentiable. For any $v \in \Omega_{p}(V)$, let $z_{n}:=I_{n}^{\prime}(x) v$. Then

$$
z_{n+1}=\int_{0}^{t} f^{\prime}\left(y_{n}(s)\right) z_{n}(s) d x(s)+\int_{0}^{t} f\left(y_{n}(s)\right) d v(s),
$$

with

$$
\begin{gathered}
\left\|\int_{0} f\left(y_{n}\right) d v\right\|_{[s, t], p} \leqslant\left[\xi(1 / p+\alpha / p)\|f\|_{C^{0, \alpha}}\left\|y_{n}\right\|_{[s, t], p}^{\alpha}+\|f\|_{\infty}\right]\|v\|_{[s, t], p}, \\
\left\|\int_{0} f^{\prime}\left(y_{n}\right) z_{n} d x\right\|_{[s, t], p} \leqslant\left[\xi(1 / p+\alpha / p)\left\|f^{\prime}\right\|_{C^{0, \alpha}}\left\|y_{n}\right\|_{p}^{\alpha}+\left\|f^{\prime}\right\|_{\infty}\right] \\
\times\left[\xi(2 / p)\left\|z_{n}\right\|_{[s, t],, p}+\left\|z_{n}\right\|_{\infty}\right]\|x\|_{[s, t], p} .
\end{gathered}
$$

Moreover, if for some constant $A \in(0,1)$, one has the inequality

$$
\xi(2 / p)\left[\xi(1 / p+\alpha / p)\left\|f^{\prime}\right\|_{C^{0, \alpha}} \sup _{n}\left\|y_{n}\right\|_{[s, t], p}^{\alpha}+\left\|f^{\prime}\right\|_{\infty}\right]\|x\|_{[s, t], p}<A,
$$

then there is a constant $C=C\left(\|f\|_{C^{0, \alpha}}, p, \alpha, A\right)$ such that one has the uniform a priori bound

$$
\sup _{n \in \mathbb{N}}\left\|z_{n}\right\|_{[s, t], p}<C\|v\|_{[s, t], p} .
$$

Proof. - By definition, $I_{n+1}(x)=a+\Phi\left(x, I_{n}(x)\right)$, where $\Phi(x, y)=\int_{0}^{\cdot} f(y) d x$. By induction and by Theorem 2.17, $I_{n}$ is differentiable in the sense of Fréchet and $z_{n}=I_{n}^{\prime}(x) v$ satisfies the recursive formula (2.9). The first and the second a priori estimates follow from the Young-Love inequality. Moreover, by (2.9) and these estimates, we obtain

$$
\left\|z_{n+1}\right\|_{[s, t], p} \leqslant A_{n}\left\|z_{n}\right\|_{[s, t], p}+B_{n}
$$

where

$$
\begin{aligned}
& A_{n}=\xi(2 / p)\left[\xi(1 / p+\alpha / p)\left\|f^{\prime}\right\|_{C^{0, \alpha}}\left\|y_{n}\right\|_{[s, t], p}^{\alpha}+\left\|f^{\prime}\right\|_{\infty}\right]\|x\|_{[s, t], p}, \\
& B_{n}=\left[\xi(1 / p+\alpha / p)\|f\|_{C^{0, \alpha}}\left\|y_{n}\right\|_{[s, t], p}^{\alpha}+\|f\|_{\infty}\right]\|v\|_{[s, t], p} .
\end{aligned}
$$

By [17], we see that $y_{n}$ converges in $\Omega_{p}$ to $y$, which is the solution of the differential equation $d y_{t}=f\left(y_{t}\right) d x_{t}$ with the initial condition $y_{0}=a$. Thus $\left\|y_{n}\right\|_{[s, t], p}$ is bounded by a constant $M=M\left(\|f\|_{C^{0, \alpha}}, p, \alpha, K\right)$ for all $x$ with $p$-variation less than $K$ :

$$
\sup _{n \in \mathbb{N}}\left\|y_{n}\right\|_{[s, t], p} \leqslant M
$$

This also yields that $B_{n} \leqslant C\|v\|_{p}$ for some constant $C=C\left(\|f\|_{C^{0, \alpha}}, p, \alpha, A\right)$. Hence under the condition (2.10) we have $A_{n} \leqslant A<1$, therefore

$$
\left\|z_{n+1}\right\|_{[s, t], p} \leqslant A\left\|z_{n}\right\|_{[s, t], p}+C\|v\|_{p} .
$$

$4^{\text {e }}$ SÉRIE - TOME $39-2006-\mathrm{N}^{\circ} 4$ 
By induction, and since $z_{0}=0$ we have

$$
\begin{aligned}
\left\|z_{n}\right\|_{[s, t], p} & \leqslant A^{n}\left\|z_{0}\right\|_{[s, t], p}+\sum_{i=0}^{n} A^{i} C\|v\|_{p} \\
& \leqslant \sum_{i=0}^{n} A^{i} C\|v\|_{p} .
\end{aligned}
$$

Therefore

$$
\sup _{n \in \mathbb{N}}\left\|z_{n}\right\|_{[s, t], p} \leqslant \frac{C}{1-A}\|v\|_{p} .
$$

Now we are ready to state our main result in this section.

THEOREM 2.19. - Under the same assumptions as in Theorem 2.18, for any $A \in(0,1)$, and for all $x$ with

$$
\|x\|_{[s, t], p} \leqslant \frac{A}{2}\left[\xi(2 / p)\left(\xi(1 / p+\alpha / p)\left\|f^{\prime}\right\|_{0, \alpha} M^{\alpha}+\left\|f^{\prime}\right\|_{\infty}\right)\right]^{-1}
$$

the sequence $\left(z_{n}\right)$ converges in $\Omega_{p}$ with respect to $\|\cdot\|_{[s, t], p}$. If $z$ is the limit

$$
z=\lim _{n \rightarrow \infty} z_{n} \quad \text { in } \Omega_{p}
$$

then

$$
z=\int_{0}^{t} f^{\prime}(y(s)) z(s) d x(s)+\int_{0}^{t} f(y(s)) d v(s)
$$

and for the some constant $C=C\left(\left(\|f\|_{C^{1, \alpha+\varepsilon}}, p, \alpha, A\right)\right)$

$$
\|z\|_{p} \leqslant C\|v\|_{p} .
$$

Moreover,

$$
\begin{aligned}
&\left\|\int_{0} f(y) d v\right\|_{[s, t], p} \leqslant {\left[\xi(1 / p+\alpha / p)\|f\|_{C^{0, \alpha}}\|y\|_{[s, t], p}^{\alpha}+\|f\|_{\infty}\right]\|v\|_{[s, t], p}, } \\
&\left\|\int_{0} f^{\prime}(y) z d x\right\|_{[s, t], p} \leqslant {\left[\xi(1 / p+\alpha / p)\left\|f^{\prime}\right\|_{C^{0, \alpha}}\|y\|_{[s, t], p}^{\alpha}+\left\|f^{\prime}\right\|_{\infty}\right] } \\
& \times\left[\xi(2 / p)\|z\|_{[s, t], p}+\|z\|_{\infty}\right]\|x\|_{[s, t], p} .
\end{aligned}
$$

Proof. - By (2.9), we have $z_{n+1}-z_{n}=I_{1}+I_{2}+I_{3}$, where

$$
\begin{aligned}
I_{1} & =\int_{0}^{t}\left[f\left(y_{n}(r)\right)-f\left(y_{n-1}(r)\right)\right] d v(r), \\
I_{2} & =\int_{0}^{t} f^{\prime}\left(y_{n-1}(r)\right)\left[z_{n}(r)-z_{n-1}(r)\right] d x(r),
\end{aligned}
$$

ANNALES SCIENTIFIQUES DE L'ÉCOLE NORMALE SUPÉRIEURE 


$$
I_{3}=\int_{0}^{t}\left[f^{\prime}\left(y_{n}(r)\right)-f^{\prime}\left(y_{n-1}(r)\right)\right] z_{n}(r) d x(r) .
$$

By the Young-Love inequality, Corollary 2.8 and Theorem 2.13, we have

$$
\begin{aligned}
& \left\|I_{1}\right\|_{[s, t], p}=\left\|\int_{0}\left[f\left(y_{n}(r)\right)-f\left(y_{n-1}(r)\right)\right] d v(r)\right\|_{[s, t], p} \\
& \leqslant \xi(1 / p+\alpha / p)\left\|f\left(y_{n}\right)-f\left(y_{n-1}\right)\right\|_{[s, t], p / \alpha}\|v\|_{[s, t], p} \\
& +\left\|f\left(y_{n}\right)-f\left(y_{n-1}\right)\right\|_{\infty}\|v\|_{[s, t], p} \\
& \leqslant C_{1} \xi(1 / p+\alpha / p)\|f\|_{\alpha+\varepsilon}\left\|y_{n}-y_{n-1}\right\|_{[s, t], p / \alpha}^{\frac{\varepsilon}{1+\varepsilon}}\|v\|_{[s, t], p} \\
& +\left\|f^{\prime}\right\|_{\infty}\left\|y_{n}-y_{n-1}\right\|_{\infty}\|v\|_{[s, t], p} \\
& \left\|I_{2}\right\|_{[s, t], p}=\left\|\int_{0} f^{\prime}\left(y_{n}(r)\right)\left[z_{n}(r)-z_{n-1}(r)\right] d x(r)\right\|_{[s, t], p} \\
& \leqslant\left[\xi(1 / p+\alpha / p)\left\|f^{\prime}\right\|_{0, \alpha}\left\|y_{n}\right\|_{[s, t], p}^{\alpha}+\left\|f^{\prime}\right\|_{\infty}\right]\left\|\int_{0}\left[z_{n}(r)-z_{n-1}(r)\right] d x(r)\right\|_{[s, t], p} \\
& \leqslant\left[\xi(1 / p+\alpha / p)\left\|f^{\prime}\right\|_{0, \alpha}\left\|y_{n}\right\|_{[s, t], p}^{\alpha}+\left\|f^{\prime}\right\|_{\infty}\right] \\
& \times\left[\xi(2 / p)\left\|z_{n}-z_{n-1}\right\|_{[s, t], p}+\left\|z_{n}-z_{n-1}\right\|_{\infty}\right]\|x\|_{[s, t], p} ; \\
& \left\|I_{3}\right\|_{[s, t], p}=\left\|\int_{0}\left[f^{\prime}\left(y_{n}(r)\right)-f^{\prime}\left(y_{n-1}(r)\right)\right] z_{n}(r) d v(r)\right\|_{[s, t], p} \\
& \leqslant \xi(1 / p+\alpha / p)\left\|f^{\prime}\left(y_{n}\right)-f^{\prime}\left(y_{n-1}\right)\right\|_{[s, t], p / \alpha}\left\|\int_{0} z_{n}(r) d x(r)\right\|_{[s, t], p} \\
& +\left\|f^{\prime}\left(y_{n}\right)-f^{\prime}\left(y_{n-1}\right)\right\|_{\infty}\left\|\int_{0} z_{n}(r) d x(r)\right\|_{[s, t], p} \\
& \leqslant\left[C_{1} \xi(1 / p+\alpha / p)\left\|f^{\prime}\right\|_{\alpha+\varepsilon}\left\|y_{n}-y_{n-1}\right\|_{[s, t], p}^{\varepsilon /(1+\varepsilon)}+\left\|f^{\prime}\right\|_{\alpha}\left\|y_{n}-y_{n-1}\right\|_{\infty}^{\alpha}\right] \\
& \times\left[\xi(2 / p)\left\|z_{n}\right\|_{[s, t], p}+\left\|z_{n}\right\|_{\infty}\right]\|x\|_{[s, t], p} .
\end{aligned}
$$

Hence

$$
\begin{aligned}
\left\|z_{n+1}-z_{n}\right\|_{[s, t], p} & \leqslant A_{n}\left[\left\|z_{n}-z_{n-1}\right\|_{[s, t], p}+\left\|z_{n}-z_{n-1}\right\|_{\infty}\right]+B_{n} \\
& \leqslant 2 A_{n}\left\|z_{n}-z_{n-1}\right\|_{[s, t], p}+B_{n} .
\end{aligned}
$$

Here

$$
\begin{aligned}
A_{n}= & \xi(2 / p)\left[\xi(1 / p+\alpha / p)\left\|f^{\prime}\right\|_{0, \alpha}\left\|y_{n}\right\|_{[s, t], p}^{\alpha}+\left\|f^{\prime}\right\|_{\infty}\right]\|x\|_{[s, t], p}, \\
B_{n}= & \xi(1 / p+\alpha / p) C_{1}\|f\|_{\alpha+\varepsilon}\left\|y_{n}-y_{n-1}\right\|_{[s, t], p}^{\frac{\varepsilon}{1+\varepsilon}}\|v\|_{[s, t], p} \\
& +\left\|f^{\prime}\right\|_{\infty}\left\|y_{n}-y_{n-1}\right\|_{\infty}\|v\|_{[s, t], p}
\end{aligned}
$$




$$
\begin{aligned}
& +\left[\xi(1 / p+\alpha / p)\left\|f^{\prime}\right\|_{\alpha+\varepsilon}\left\|y_{n}-y_{n-1}\right\|_{[s, t], p}^{\frac{\varepsilon}{1+\varepsilon}}+\left\|f^{\prime}\right\|_{\alpha}\left\|y_{n}-y_{n-1}\right\|_{\infty}^{\alpha}\right] \\
& \times\left[\xi(2 / p)\left\|z_{n}\right\|_{[s, t], p}+\left\|z_{n}\right\|_{\infty}\right]\|x\|_{[s, t], p} .
\end{aligned}
$$

Since

$$
\sup _{n \in \mathbb{N}}\left\|y_{n}\right\|_{[s, t], p} \leqslant M
$$

we have

$$
A_{n} \leqslant \xi(2 / p)\left[\xi(1 / p+\alpha / p)\left\|f^{\prime}\right\|_{0, \alpha} M^{\alpha}+\left\|f^{\prime}\right\|_{\infty}\right]\|x\|_{[s, t], p} .
$$

Thus, for any $A \in(0,1)$ and for all $x$ such that

$$
\|x\|_{[s, t], p} \leqslant \frac{A}{2}\left[\xi(2 / p)\left(\xi(1 / p+\alpha / p)\left\|f^{\prime}\right\|_{0, \alpha} M^{\alpha}+\left\|f^{\prime}\right\|_{\infty}\right)\right]^{-1}
$$

we have

$$
\left\|z_{n+1}-z_{n}\right\|_{[s, t], p} \leqslant A\left\|z_{n}-z_{n-1}\right\|_{[s, t], p}+2 B_{n} .
$$

By induction, and since $z_{0}=0$,

$$
\left\|z_{n+1}-z_{n}\right\|_{[s, t], p} \leqslant A^{n}\left\|z_{1}\right\|_{[s, t], p}+2 \sum_{i=0}^{n} A^{i} B_{n-i} .
$$

Hence

$$
\begin{aligned}
\sum_{n=0}^{\infty}\left\|z_{n+1}-z_{n}\right\|_{[s, t], p} & \leqslant \frac{A}{1-A}\left\|z_{1}\right\|_{[s, t], p}+2 \sum_{n=0}^{\infty} \sum_{i=0}^{n} A^{i} B_{n_{i}} \\
& =\frac{A}{1-A}\left\|z_{1}\right\|_{[s, t], p}+2 \sum_{n=0}^{\infty} A^{i} \sum_{i=0}^{\infty} B_{n} \\
& =\frac{A}{1-A}\left\|z_{1}\right\|_{p}+\frac{2 A}{1-A} \sum_{n=0}^{\infty} B_{n} .
\end{aligned}
$$

To show that $\left(z_{n}\right)$ converges in $\Omega_{p}$ we need only to show that $\sum_{n=0}^{\infty} B_{n}<+\infty$.

By Theorem 2.18, we have $\sup _{n \in \mathbb{N}}\left\|z_{n}\right\|_{[s, t], p, \infty}<C_{2}\|v\|_{p}$. Moreover, from the explicit expression for $B_{n}$, we have

$$
B_{n} \leqslant C_{1}\left\|y_{n}-y_{n-1}\right\|_{[s, t], p}^{\frac{\varepsilon}{1+\varepsilon}}\|v\|_{p}+C_{2}\left\|y_{n}-y_{n-1}\right\|_{\infty}^{\alpha}\|v\|_{p}+C_{3}\left\|y_{n}-y_{n-1}\right\|_{\infty}\|v\|_{p},
$$

where $C_{1}=C\|f\|_{1+\alpha+\varepsilon}, C_{2}=C\|f\|_{1+\alpha}, C_{3}=C\|f\|_{1}$ and

$$
C=C\left(\|f\|_{1+\alpha+\varepsilon}, \alpha, \varepsilon, p, A\right) .
$$

Let $\beta=\min \left\{\alpha, \frac{\varepsilon}{1+\varepsilon}\right\}$. Then

$$
\begin{aligned}
B_{n} & \leqslant C\|f\|_{1+\alpha+\varepsilon}\|v\|_{p}\left[\left\|y_{n}-y_{n-1}\right\|_{p}^{\frac{\varepsilon}{1+\varepsilon}}+\left\|y_{n}-y_{n-1}\right\|_{p}^{\alpha}+\left\|y_{n}-y_{n-1}\right\|_{p}\right] \\
& \leqslant C\|f\|_{1+\alpha+\varepsilon}\left\|y_{n}-y_{n-1}\right\|_{p}^{\beta}\left[1+\left\|y_{n}-y_{n-1}\right\|_{p}^{\alpha-\frac{\varepsilon}{1+\varepsilon}}+\left\|y_{n}-y_{n-1}\right\|_{p}^{1-\beta}\right] \\
& \leqslant C\|f\|_{1+\alpha+\varepsilon}\left\|y_{n}-y_{n-1}\right\|_{p}^{\beta}\left[1+\sup _{k \in \mathbb{N}}\left\|y_{k}-y_{k-1}\right\|_{p}^{\alpha-\frac{\varepsilon}{1+\varepsilon}}+\sup _{k \in \mathbb{N}}\left\|y_{k}-y_{k-1}\right\|_{p}^{1-\beta}\right] .
\end{aligned}
$$


Following [17, p. 461], there exists a constant $C_{4}$ such that

$$
\left\|y_{n}-y_{n-1}\right\|_{[s, t], p} \leqslant[1+\xi(2 / p)]^{n-1} C_{4}^{n}\|f\|_{1+\alpha}^{n}\left[\frac{\|x\|_{[0, t], p}^{n}-\|x\|_{[0, s], p}^{n}}{n !}\right]^{1 / p} .
$$

Hence, there exists a constant $C_{5}$ such that

$$
B_{n} \leqslant C_{5}\|f\|_{1+\alpha+\varepsilon}\left([1+\xi(2 / p)] C_{4}\|f\|_{1+\alpha}\|x\|_{[0,1], p}^{1 / p}\right)^{n \beta}(n !)^{-\beta / p} .
$$

Therefore for an appropriate constant $C$ we have

$$
\begin{aligned}
\sum_{n=0}^{\infty} B_{n} & \leqslant C_{5}\|f\|_{1+\alpha+\varepsilon} \sum_{n=1}^{\infty} \frac{C^{n}}{(n !)^{\beta / p}} \\
& \leqslant C_{5}\|f\|_{1+\alpha+\varepsilon}\left(C+\frac{1}{C}\right) \frac{p}{\beta} e^{C^{\frac{p}{\beta}}}
\end{aligned}
$$

since $(n !)^{\beta / p}>[n \beta / p]$ !

Now we come back to the proof of Theorem 2.19. The convergence of $\left(z_{n}\right)$ in $\left(\Omega_{p},\|\cdot\|_{[s, t], p}\right)$ follows from $\sum_{n=0}^{\infty} B_{n}<+\infty$. Using the continuity of the Young integral (see [18,22]) and (2.9), it is easy to see that $z=\lim _{n \rightarrow \infty} z_{n}$ satisfies (2.12). The lower-semi-continuity of the norm $\|\cdot\|_{p}$ and the last inequality in Theorem 2.18 yield the uniform bound $\|z\|_{p} \leqslant C\|v\|_{p}$. The a priori estimates in Theorem 2.18 follow from the Young-Love inequality.

\subsection{Lipschitz continuity of the derivatives of the Itô map}

In this section we shall prove the Lipschitz continuity of the derivative of the Itô map under the appropriate smoothness assumption on $f$.

THEOREM 2.20. - Let $f \in \operatorname{Lip}(1+\alpha+\varepsilon)$ with $\alpha \in(p-1,1-\varepsilon)$ and $M$ (the same constant appeared in the proof of Theorem 2.18, see [17]) be such that $\sup _{n \in \mathbb{N}}\left\|y_{n}\right\|_{[s, t], p} \leqslant M$. Let $K$ be chosen so that

$$
K \leqslant A\left[\left(\xi(1 / p+\alpha / p)\left\|f^{\prime}\right\|_{0, \alpha} M^{\alpha}+\left\|f^{\prime}\right\|_{\infty}\right)\right]^{-1}, \quad 0<A<1 .
$$

Then for all $x, \tilde{x} \in \Omega_{p}(V)$ with $\|x\|_{p} \leqslant K$ and $\|\tilde{x}\|_{p} \leqslant K$ and for all $v \in \Omega_{p}(V)$,

$$
\|z-\tilde{z}\| \leqslant C\|x-\tilde{x}\|_{p}^{\frac{\varepsilon}{1+\varepsilon}}\|v\|_{p}
$$

where $z=I^{\prime}(x) v, \tilde{z}=I^{\prime}(\tilde{x}) v$.

Proof. - Let $y=I(x), \tilde{y}=I(\tilde{x})$. By Theorem 2.19, we have

$$
\begin{array}{ll}
d z=f^{\prime}(y) z d x+f(y) d v, & z_{0}=v, \\
d \tilde{z}=f^{\prime}(\tilde{y}) \tilde{z} d \tilde{x}+f(\tilde{y}) d v, & \tilde{z}_{0}=v .
\end{array}
$$

Hence 


$$
\begin{aligned}
{[z-\tilde{z}]_{s t}=} & \int_{s}^{t} f^{\prime}(y)[z-\tilde{z}] d x+\int_{s}^{t} f^{\prime}(\tilde{y}) \tilde{z} d(x-\tilde{x}) \\
& +\int_{s}^{t}[f(y)-f(\tilde{y})] d v+\int_{s}^{t}\left[f^{\prime}(y)-f^{\prime}(\tilde{y})\right] \tilde{z} d x .
\end{aligned}
$$

Using the Young-Love inequality, we can obtain

$$
\begin{aligned}
\|z-\tilde{z}\|_{p} \leqslant & {\left[\xi(1 / p+\alpha / p)\left\|f^{\prime}\right\|_{\alpha} n^{\alpha}+\left\|f^{\prime}\right\|_{\infty}\right]\|z-\tilde{z}\|_{p}\|x\|_{p} } \\
& +C\left\|f^{\prime}\right\|_{\alpha}\|y\|_{p}^{\alpha}\|z\|_{p}\|x-\tilde{x}\|_{p}+C\|f\|_{1+\alpha}\|y-\tilde{y}\|_{p}\|v\|_{p} \\
& +C\left\|f^{\prime}\right\|_{\alpha+\varepsilon}\|y-\tilde{y}\|_{p}^{\frac{\varepsilon}{1+\varepsilon}}\|\tilde{z}\|_{p}\|x\|_{p},
\end{aligned}
$$

where we have used Theorem 2.13 for the estimate of $\left\|f^{\prime}(y)-f^{\prime}(\tilde{y})\right\|_{\frac{p}{\alpha}}$. Therefore

$$
\|z-\tilde{z}\|_{p} \leqslant A\|z-\tilde{z}\|_{p}+B
$$

Here

$$
\begin{aligned}
A & =\left[\xi(1 / p+\alpha / p)\left\|f^{\prime}\right\|_{\alpha} n^{\alpha}+\left\|f^{\prime}\right\|_{\infty}\right]\|x\|_{p}, \\
B & =C\|f\|_{1+\alpha+\varepsilon}\left[\|x\|_{p}\|\tilde{z}\|_{p}\|y-\tilde{y}\|_{p}^{\frac{\varepsilon}{1+\varepsilon}}+\|y\|_{p}^{\alpha}\|z\|_{p}\|x-\tilde{x}\|_{p}+\|v\|_{p}\|y-\tilde{y}\|_{p}\right] \\
& \leqslant C\|f\|_{1+\alpha+\varepsilon}\left[\|x\|_{p}+\|y\|_{p}^{\alpha}\right]\left[\|x-\tilde{x}\|_{p}+\|y-\tilde{y}\|_{p}+\|y-\tilde{y}\|_{p}^{\frac{\varepsilon}{1+\varepsilon}}\right]\|v\|_{p} .
\end{aligned}
$$

Using the Lipschitz continuity of the Itô map, cf. [18,22], we have

$$
\|y-\tilde{y}\|_{p} \leqslant C\|x-\tilde{x}\|_{p} .
$$

Modifying the proof of Theorem 2.18, we can also prove that for $\|x\|_{p} \leqslant K$ and $\|\tilde{x}\|_{p} \leqslant K$, we have

$$
\|z-\tilde{z}\|_{p} \leqslant C_{K}\|f\|_{1+\alpha+\varepsilon}\|x-\tilde{x}\|_{p}^{\frac{\varepsilon}{1+\varepsilon}}\|v\|_{p}
$$

Now we are ready to prove Theorem 2.2 concerning the Fréchet differentiability of the Itô map.

Proof of Theorem 2.2. - By the main result of [17], $I_{n}$ converges uniformly to $I$ and $I$ is continuous from the $p$-variation ball $B_{p}(0, K) \subset \Omega_{p}(V)$ into $\Omega_{p}(W)$. Moreover, Theorem 2.19 says that the derivatives $z_{n}=I_{n}^{\prime}(x) v$ converge uniformly to $z=z(x)$ in $\Omega_{p}(W)$, and Theorem 2.20 says that $x \rightarrow z(x)$ is $\frac{\varepsilon}{1+\varepsilon}$-Hölder continuous on $B_{p}(0, K)=\left\{x \in V:\|x\|_{p} \leqslant K\right\}$. We conclude that $I$ is locally Fréchet differentiable ${ }^{3}$ from the ball $B_{p}(0, K) \subset \Omega_{p}(V)$ into $\Omega_{p}(W)$. Moreover, its derivative is given by

$$
I^{\prime}(x) v=z .
$$

Furthermore, Theorem 2.20 can be reformulated as

$$
\left\|I^{\prime}(x)-I^{\prime}(\tilde{x})\right\| \leqslant C\|x-\tilde{x}\|_{p}^{\frac{\varepsilon}{1+\varepsilon}} .
$$

\footnotetext{
${ }^{3}$ If $f_{n}: E_{1} \rightarrow E_{2}$ is a sequence of continuously Fréchet differentiable (in the sense of) functions between two Banach spaces such that $f_{n} \rightarrow f$ and their derivatives $f_{n}^{\prime} \in L\left(E_{1}, E_{2}\right)$ are uniformly convergent with continuous limit $g \in L\left(E_{1}, E_{2}\right)$, then $f$ is Fréchet differentiable and $f^{\prime}=g$.
} 
This proves the $\frac{\varepsilon}{1+\varepsilon}$-Hölder continuity of the derivative of the Itô map.

THEOREM 2.21. - Let $f \in \operatorname{Lip}(1+\alpha+\varepsilon)$ with $\alpha \in(p-1,1-\varepsilon)$. Then, defining $K$ as in Theorem 2.20, one has that for any $x, v \in \Omega_{p}(V)$ with $\|x\|_{p} \leqslant K$ and $\|v\|_{p} \leqslant K$ :

$$
\left\|I(x+v)-I(x)-I^{\prime}(x) v\right\|_{p} \leqslant C\|f\|_{1+\alpha+\varepsilon}\|v\|_{p}^{\frac{1+2 \varepsilon}{1+\varepsilon}},
$$

where $C=C\left(K, \alpha, p,\|f\|_{1+\alpha}\right)$ is a constant.

Proof. - Denote $\tilde{x}=x+v$. Then

$$
\begin{aligned}
& d y_{t}=f\left(y_{t}\right) d x_{t}, \quad y_{0}=a, \\
& d \tilde{y}_{t}=f\left(\tilde{y}_{t}\right) d \tilde{x}_{t}, \quad \tilde{y}_{0}=a, \\
& d z_{t}=f^{\prime}\left(y_{t}\right) z_{t} d x_{t}+f\left(y_{t}\right) d v_{t}, \quad z_{0}=0 .
\end{aligned}
$$

Note that

$$
\begin{aligned}
d\left(\tilde{y}_{t}-y_{t}-z_{t}\right)= & f\left(\tilde{y}_{t}\right) d \tilde{x}_{t}-f\left(y_{t}\right) d x_{t}-f^{\prime}\left(y_{t}\right) z_{t} d x_{t}-f\left(y_{t}\right) d\left(\tilde{x}_{t}-x_{t}\right) \\
= & f\left(\tilde{y}_{t}\right) d \tilde{x}_{t}-f\left(y_{t}\right) d \tilde{x}_{t}-f^{\prime}\left(y_{t}\right) z_{t} d x_{t} \\
= & f^{\prime}\left(y_{t}\right) z_{t} d v_{t}+f^{\prime}\left(y_{t}\right)\left[\tilde{y}_{t}-y_{t}-z_{t}\right] d \tilde{x}_{t} \\
& +\left[f\left(\tilde{y}_{t}\right)-f\left(y_{t}\right)-f^{\prime}\left(y_{t}\right)\left(\tilde{y}_{t}-y_{t}\right)\right] d \tilde{x}_{t} .
\end{aligned}
$$

Therefore

$$
\|\tilde{y}-y-z\|_{[s, t], p} \leqslant J_{1}+J_{2}+J_{3},
$$

where

$$
\begin{aligned}
& J_{1}=\left\|\int_{0} f^{\prime}\left(y_{u}\right) z_{u} d v_{u}\right\|_{[s, t], p}, \\
& J_{2}=\left\|\int_{0} f^{\prime}\left(y_{u}\right)\left[\tilde{y}_{u}-y_{u}-z_{u}\right] d \tilde{x}_{u}\right\|_{[s, t], p}, \\
& J_{3}=\left\|\int_{0}\left[f\left(\tilde{y}_{u}\right)-f\left(y_{u}\right)-f^{\prime}\left(y_{u}\right)\left(\tilde{y}_{u}-y_{u}\right)\right] d \tilde{x}_{u}\right\|_{[s, t], p} .
\end{aligned}
$$

Using the Young-Love inequality, Theorem 2.15 and the Lipschitz continuity theorem of the Itô map [17,22], we have

$$
\begin{aligned}
J_{1} & \leqslant\left[\xi(1 / p+\alpha / p)\left\|f^{\prime}(y)\right\|_{[s, t], p}+\left\|f^{\prime}(y)\right\|_{\infty}\right]\left\|\int_{0} z_{u} d v_{u}\right\|_{[s, t], p} \\
& \leqslant\left[\xi(1 / p+\alpha / p)\|f\|_{1+\alpha}\|y\|_{[s, t], p}^{\alpha}+\left\|f^{\prime}\right\|_{\infty}\right]\|z\|_{[s, t], p}\|\tilde{x}-x\|_{[s, t], p}, \\
J_{2} & \leqslant\left[\xi(1 / p+\alpha / p)\left\|f^{\prime}(y)\right\|_{[s, t], \alpha / p}+\left\|f^{\prime}(y)\right\|_{\infty}\right]\left\|\int_{0}\left[\tilde{y}_{u}-y_{u}-z_{u}\right] d \tilde{x}_{u}\right\|_{[s, t], p} \\
& \leqslant\left[\xi(1 / p+\alpha / p)\left\|f^{\prime}(y)\right\|_{[s, t], \alpha / p}+\left\|f^{\prime}(y)\right\|_{\infty}\right]
\end{aligned}
$$

$4^{\text {e }}$ SÉRIE - TOME $39-2006-\mathrm{N}^{\circ} 4$ 


$$
\begin{aligned}
& \times\left[\xi(2 / p)\|\tilde{y}-y-z\|_{[s, t], p}+\|\tilde{y}-y-z\|_{\infty}\right]\|\tilde{x}\|_{[s, t], p} \\
\leqslant & \|\tilde{x}\|_{[s, t], p}(1+\xi(2 / p)) \\
& \times\left[\xi(1 / p+\alpha / p)\|f\|_{1+\alpha}\|y\|_{[s, t], p}^{\alpha}+\left\|f^{\prime}\right\|_{\infty}\right]\|\tilde{y}-y-z\|_{[s, t], p}, \\
J_{3} \leqslant & {[1+\xi(1 / p+\alpha / p)]\left\|f(\tilde{y})-f(y)-f^{\prime}(y)(\tilde{y}-y)\right\|_{[s, t], p}\|\tilde{x}\|_{[s, t], p} } \\
\leqslant & C\left(\alpha, p,\|f\|_{1+\alpha+\varepsilon}\right)\|y-\tilde{y}\|_{p}^{\frac{1+2 \varepsilon}{1+\varepsilon}}\|\tilde{x}\|_{[s, t], p} \\
\leqslant & C_{2}\left(\alpha, p,\|f\|_{1+\alpha+\varepsilon}\right) K\|\tilde{x}-x\|_{[s, t], p}^{\frac{1+2 \varepsilon}{1+\varepsilon}} .
\end{aligned}
$$

Note that by Theorem 2.18 and the lower-semi-continuity of the norm $\|\cdot\|_{p}$, we have

$$
\|z\|_{[s, t], p} \leqslant C_{3}\|v\|_{[s, t], p}=C_{3}\|\tilde{x}-x\|_{[s, t], p} .
$$

Thus

$$
\begin{aligned}
\|\tilde{y}-y-z\|_{[s, t], p} \leqslant & \|\tilde{x}\|_{[s, t], p}\left[\xi(1 / p+\alpha / p)\|f\|_{1+\alpha}\|y\|_{[s, t], p}^{\alpha}+\left\|f^{\prime}\right\|_{\infty}\right] \\
& \times\|\tilde{y}-y-z\|_{[s, t], p}+C_{2}\left(\alpha, p,\|f\|_{1+\alpha+\varepsilon}, K\right)\|\tilde{x}-x\|_{[s, t], p}^{\frac{1+2 \varepsilon}{1+\varepsilon}} \\
& +C_{4}\left(\alpha, p,\|f\|_{1+\alpha}, K\right)\|\tilde{x}-x\|_{[s, t], p}^{2} .
\end{aligned}
$$

Hence, for $x, \tilde{x} \in \Omega_{p}\left(\mathbb{R}^{m}\right)$ with $\|x\|_{p} \leqslant K,\|\tilde{x}\|_{p} \leqslant K$, we have

$$
\|\tilde{y}-y-z\|_{[s, t], p} \leqslant A\|\tilde{y}-y-z\|_{[s, t], p}+C_{2}\|\tilde{x}-x\|_{[s, t], p}^{\frac{1+2 \varepsilon}{1+\varepsilon}}+C_{4}\|\tilde{x}-x\|_{[s, t], p}^{2} .
$$

Hence

$$
\begin{aligned}
\|\tilde{y}-y-z\|_{[s, t], p} \leqslant & (1-A)^{-1} C_{2}\|f\|_{1+\alpha+\varepsilon}\|\tilde{x}-x\|_{[s, t], p}^{\frac{1+2 \varepsilon}{1+\varepsilon}} \\
& +(1-A)^{-1} C_{4}\|f\|_{1+\alpha}\|\tilde{x}-x\|_{[s, t], p}^{2} \\
\leqslant & C_{5}\left(K, \alpha, p,\|f\|_{1+\alpha+\varepsilon}\right)\|f\|_{1+\alpha+\varepsilon}\|\tilde{x}-x\|_{[s, t], p}^{\frac{1+2 \varepsilon}{1+\varepsilon}}
\end{aligned}
$$

In the special case where $f \in \operatorname{Lip}(2+\alpha)$ we have the following estimate.

THEOREM 2.22. - Let $f \in \operatorname{Lip}(2+\alpha)$. Then for $x, \tilde{x} \in B(0, K)$, we have

$$
\begin{array}{r}
\left\|I^{\prime}(x)-I^{\prime}(\tilde{x})\right\|_{p} \leqslant C_{6}\left(\alpha,\|f\|_{2+\alpha}, K\right)\|x-\tilde{x}\|_{p}, \\
\left\|I(\tilde{x})-I(x)-I^{\prime}(x)(\tilde{x}-x)\right\|_{p} \leqslant C_{7}\left(\alpha,\|f\|_{2, \alpha}, K\right)\|x-\tilde{x}\|_{p}^{2} .
\end{array}
$$

Proof. - By Lemma 2.12 and the Young-Love inequality we have

$$
\begin{aligned}
& \left\|\int_{0}\left[f^{\prime}(y)-f^{\prime}(\tilde{y})\right] \tilde{z} d x\right\|_{p} \\
& \quad \leqslant\left[\xi(1 / p+\alpha / p)\left\|f^{\prime}(y)-f^{\prime}(\tilde{y})\right\|_{p / \alpha}+\left\|f^{\prime}(y)-f^{\prime}(\tilde{y})\right\|_{\infty}\right]\|\tilde{z}\|_{p}\|x\|_{p} \\
& \quad \leqslant\left[\xi(1 / p+\alpha / p) C\left(\alpha, K,\left\|f^{\prime}\right\|_{1+\alpha}\right)\|y-\tilde{y}\|_{p}+\left\|f^{\prime \prime}\right\|_{\infty}\|y-\tilde{y}\|_{\infty}\right]\|z\|_{p}\|x\|_{p} \\
& \quad \leqslant C\left(\alpha, K,\|f\|_{2+\alpha}\right)\|v\|_{p}\|y-\tilde{y}\|_{p} .
\end{aligned}
$$

From this and the proof of Theorem 2.20 we have

$$
\|z-\tilde{z}\|_{p} \leqslant A\|z-\tilde{z}\|_{p}+C\left(\alpha, K,\|f\|_{2+\alpha}\right)\left[\|x-\tilde{x}\|_{p}+\|y-\tilde{y}\|_{p}\right]\|v\|_{p} .
$$


Hence for $\|x\|_{p} \leqslant K$ and $\|\tilde{x}\|_{p} \leqslant K$ we have

$$
\|z-\tilde{z}\|_{p} \leqslant C\|f\|_{2+\alpha}\|v\|_{p}\left[\|x-\tilde{x}\|_{p}+\|y-\tilde{y}\|_{p}\right] .
$$

Using the Lipschitz continuity of the Itô map, we obtain

$$
\|z-\tilde{z}\|_{p} \leqslant C\|f\|_{2+\alpha}\|v\|_{p}\|x-\tilde{x}\|_{p}
$$

This is equivalent to

$$
\left\|I^{\prime}(x)-I^{\prime}(\tilde{x})\right\|_{p} \leqslant C\left(\alpha, K,\|f\|_{2+\alpha}\right)\|x-\tilde{x}\|_{p}
$$

Similarly, using Lemma 2.14 and the Young-Love inequality we can prove

$$
\begin{aligned}
J_{2} \leqslant & \xi(1 / p+\alpha / p)\left\|f(\tilde{y})-f(y)-f^{\prime}(y)(\tilde{y}-y)\right\|_{p}\|\tilde{x}\|_{p} \\
& +\left\|f(\tilde{y})-f(y)-f^{\prime}(y)(\tilde{y}-y)\right\|_{\infty}\|\tilde{x}\|_{p} \\
\leqslant & {\left[C\left(\alpha, p,\|f\|_{2+\alpha}\right) \xi(1 / p+\alpha / p)\|y-\tilde{y}\|_{p}^{2}+\left\|f^{\prime \prime}\right\|_{\infty}\|y-\tilde{y}\|_{\infty}^{2}\right]\|\tilde{x}\|_{[s, t], p} } \\
\leqslant & C\left(\alpha, p, K,\|f\|_{2+\alpha}\right) K\|y-\tilde{y}\|_{p}^{2} \\
\leqslant & C\left(\alpha, p, K,\|f\|_{2+\alpha}\right)\|x-\tilde{x}\|_{p}^{2} .
\end{aligned}
$$

From this and the proof of Theorem 2.21 we deduce that

$$
\|\tilde{y}-y-z\|_{[s, t], p} \leqslant A\|\tilde{y}-y-z\|_{[s, t], p}+C\|\tilde{x}-x\|_{[s, t], p}^{2} .
$$

Hence for $\|x\| \leqslant K$ and $\|\tilde{x}\|_{p} \leqslant K$ we get

$$
\|\tilde{y}-y-z\|_{[s, t], p} \leqslant C\left(K, \alpha, p,\|f\|_{2+\alpha}\right)\|\tilde{x}-x\|_{p}^{2} .
$$

\subsection{Higher order differentiability of the Itô map}

By induction, for any $k \in \mathbb{N}$, a map $f: E_{1} \rightarrow E_{2}$ between two Banach spaces $E_{1}$ and $E_{2}$ is $k$-times differentiable at $x_{0} \in E_{1}$ in the sense of Fréchet if there exist a constant $K>0$ and a bounded linear map $f^{(k)}\left(x_{0}\right) \in L_{k}\left(E_{1}, E_{2}\right):=L\left(E_{1}, L_{k-1}\left(E_{1}, E_{2}\right)\right)$ such that for $v \in B(0, K)$,

$$
\left\|f^{(k-1)}\left(x_{0}+v\right)-f^{(k-1)}\left(x_{0}\right)-f^{(k)}\left(x_{0}\right) v\right\|_{L_{k-1}\left(E_{1}, E_{2}\right)}=o\left(\|v\|_{E_{1}}\right) .
$$

If there exists a bounded open subset $O \subset E_{1}$ such that $f$ is $k$-times differentiable at $x_{0}$ for all $x_{0} \in O$, we call $f$ a locally $k$-times Fréchet differentiable function in $O$. We now prove the higher order Fréchet differentiability of the Itô map.

THEOREM 2.23. - Let $f \in \operatorname{Lip}(k+\alpha+\varepsilon)$ with $\alpha \in(p-1,1-\varepsilon)$. Then the Itô map $I: \Omega_{p}(V) \rightarrow \Omega_{p}(W)$ is locally $k$-times Fréchet differentiable. More precisely, there exists a constant $K$ depended on $\|f\|_{k+\alpha+\varepsilon}$ such that for $x, \tilde{x}, v \in \Omega_{p}(V)$ with $\|x\|_{p} \leqslant K$, $\|\tilde{x}\|_{p} \leqslant K$, and $\|v\|_{p} \leqslant K$, there exist bounded multilinear maps denoted by $I^{(i)}(x): \Omega_{p} \rightarrow$ $L_{i}\left(\Omega_{p}(V), \Omega_{p}(W)\right), i=1, \ldots, k$, such that

$$
\left\|I^{(k-1)}(x+v)-I^{(k-1)}(x)-I^{(k)}(x) v\right\|_{L_{k-1}\left(\Omega_{p}(V), \Omega_{p}(W)\right)} \leqslant C\left(\|f\|_{k+\alpha+\varepsilon}\right)\|v\|_{p^{\frac{1+2 \varepsilon}{1+\varepsilon}}},
$$

$4^{\mathrm{e}}$ SÉRIE - TOME $39-2006-\mathrm{N}^{\circ} 4$ 
and

$$
\left\|I^{(k)}(\tilde{x})-I^{(k)}(x)\right\|_{L_{k}\left(\Omega_{p}(V), \Omega_{p}(W)\right)} \leqslant C\left(\|f\|_{k+\alpha+\varepsilon)}\|\tilde{x}-x\|_{p}^{\frac{\varepsilon}{1+\varepsilon}} .\right.
$$

Moreover, in the case where $f \in \operatorname{Lip}(k+1+\alpha)$ with $\alpha \in(p-1,1)$, one has

$$
\left\|I^{(k-1)}(x+v)-I^{(k-1)}(x)-I^{(k)}(x) v\right\|_{L_{k-1}\left(\Omega_{p}(V), \Omega_{p}(W)\right)} \leqslant C\left(\|f\|_{k+1+\alpha}\right)\|v\|_{p}^{2},
$$

and

$$
\left\|I^{(k)}(\tilde{x})-I^{(k)}(x)\right\|_{L_{k}\left(\Omega_{p}(V), \Omega_{p}(W)\right)} \leqslant C\left(\|f\|_{k+1+\alpha}\right)\|\tilde{x}-x\|_{p}
$$

Proof. - Since the proofs for all $k$ are similar we will only give a proof for $k=2$. Let $z_{i}(x, t)=\left(I^{\prime}(x) v_{i}\right)(t), i=1,2$. Then

$$
d z_{i}=f^{\prime}(y) z_{i} d x+f(y) d v_{i}, \quad z_{i}(0)=0 .
$$

Thus

$$
z_{1}\left(x+\varepsilon v_{2}\right)=\int_{0}^{t} f^{\prime}\left(y\left(x+\varepsilon v_{2}\right)\right) z_{1}\left(x+\varepsilon v_{2}\right) d\left(x+\varepsilon v_{2}\right)+\int_{0}^{t} f\left(y\left(x+\varepsilon v_{2}\right)\right) d v_{1} .
$$

Using the same argument as used in Section 2.3, we can prove that for any $\|x\|_{p} \leqslant K$,

$$
W(x):=\lim _{\varepsilon \rightarrow 0} \frac{z_{1}\left(x+\varepsilon v_{2}\right)-z_{1}(x)}{\varepsilon}
$$

exists in $\Omega_{p}(W)$ and satisfies the following linear differential equation

$$
d W=f^{\prime}(y) W d x+f^{\prime \prime}(y)\left(z_{2} \otimes z_{1}\right) d x+f^{\prime}(y) z_{1} d v_{2}+f^{\prime}(y) z_{2} d v_{1},
$$

with the initial condition $W(0)=0$. Repeating the same argument used in the proof of Theorem 2.15, we can prove that for $\|x\|_{p} \leqslant K,\left\|v_{1}\right\|_{p} \leqslant K$, and $\left\|v_{2}\right\|_{p} \leqslant K$,

$$
\begin{gathered}
\|W(x)\|_{p} \leqslant C\left\|v_{1}\right\|_{p}\left\|v_{2}\right\|_{p}, \\
\left\|z_{1}\left(x+v_{2}\right)-z_{1}(x)-W(x)\right\|_{p} \leqslant C\left\|v_{1}\right\|\left\|_{p}\right\| v_{2} \|_{p}^{\frac{1+2 \varepsilon}{1+\varepsilon}}, \\
\|W(\tilde{x})-W(x)\|_{p} \leqslant C\|\widetilde{x}-x\|_{p}^{\frac{\varepsilon}{1+\varepsilon}}
\end{gathered}
$$

Hence, the Itô map $I: \Omega_{p}(V) \rightarrow \Omega_{p}(W)$ is locally $C^{2, \frac{\varepsilon}{1+\varepsilon}}$-differentiable in the sense of Fréchet with

$$
I^{\prime \prime}(x)\left(v_{1}, v_{2}\right)=W(x), \quad \forall x, v_{1}, v_{2} \in B_{p}(0, K) .
$$

The proof for the latter case where $f \in \operatorname{Lip}(k+1+\alpha)$ is easy so we omit it.

The following Taylor formula holds for the Itô map $I: \Omega_{p}(V) \rightarrow \Omega_{p}(W)$.

THEOREM 2.24. - Let $f \in \operatorname{Lip}(k+\alpha+\varepsilon)$ with $\alpha \in(p-1,1-\varepsilon)$. Then, for $x, v \in \Omega_{p}(V)$ with $\|x\|_{p} \leqslant K$ and $\|v\|_{p} \leqslant K$, we have

$$
I(x+v)-I(x)-\sum_{i=1}^{k-1} \frac{I^{(i)}(x) v^{\otimes i}}{i !}=\left(\int_{0}^{1} \frac{I^{(k)}(x+s v)(1-s)^{k-1}}{(k-1) !} d s\right) v^{\otimes k},
$$


and

$$
\left\|I(x+v)-I(x)-\sum_{i=1}^{k} \frac{I^{(i)}(x) v^{\otimes i}}{i !}\right\|_{p} \leqslant C\left(\|f\|_{k+\alpha+\varepsilon}\right)\|v\|_{p}^{k+\frac{\varepsilon}{1+\varepsilon}} .
$$

Moreover, in the case where $f \in \operatorname{Lip}(k+1+\alpha)$ with $\alpha \in(p-1,1)$ one has

$$
\left\|I(\tilde{x})-I(x)-\sum_{i=1}^{k} \frac{I^{(i)}(x)(\tilde{x}-x)^{\otimes i}}{i !}\right\|_{p} \leqslant C\left(p, k, \alpha, K,\|f\|_{k+1+\alpha}\right)\|\tilde{x}-x\|_{p}^{k+1} .
$$

Proof. - Let $g(t)=I(x+t v), t \in(-1-\delta, 1+\delta), \delta>0$. By the chain rule, we have $g \in C^{k, \frac{\varepsilon}{1+\varepsilon}}(-1,1)$. Applying the Taylor formula to $f$, we have

$$
g(t)=g(0)+g^{\prime}(0) t+g^{\prime \prime}(0) \frac{t^{2}}{2}+\cdots+g^{(k)}(0) \frac{t^{k-1}}{(k-1) !}+\int_{0}^{t} g^{(k)}(s) \frac{(1-s)^{k-1}}{(k-1) !} d s .
$$

Now

$$
g^{(i)}(t)=I^{(i)}(x+t v) v^{\otimes} i, \quad i=1, \ldots, k .
$$

Substituting this to the previous formula, we prove Taylor's formula with an integral remainder. Obtaining the stated estimates on this remainder is very easy.

\section{Part II — The nonlinear case}

\subsection{Path spaces on manifolds}

In this section let $M$ be a $d$-dimensional compact Riemannian manifold endowed with the Levi-Civita connection, let $m_{0} \in M$ be a fixed point and let $O(M)$ be the orthonormal frame bundle over $M$. For $1 \leqslant p<2$, let $\Omega_{p}(M)$ be the collection of $p$-variation paths on $M$ :

$$
\Omega_{p}(M)=\left\{\gamma \in C([0,1], M): \operatorname{Var}_{p}(\gamma)<\infty\right\}
$$

where

$$
\operatorname{Var}_{p}(\gamma)=\sup _{\mathcal{D}}\left(\sum_{i=1}^{n} d\left(\gamma_{s_{i}}, \gamma_{s_{i+1}}\right)^{p}\right)^{1 / p}
$$

and the supremum is taken over all finite partitions

$$
\mathcal{D}=\left\{0=s_{0}<s_{1}<\cdots<s_{n}<s_{n+1}=1\right\} .
$$

We now extend Theorem 2.2 to the nonlinear case where the driving signal $x$ and the response $y$ take values in compact Riemannian manifolds $M$ and $N$.

THEOREM 3.1. - Let $M$ and $N$ be two compact Riemannian manifolds, $f: T M \rightarrow \Gamma(T N)$ be a Lip $(k+\alpha+\varepsilon)$ bundle map ${ }^{4}, \alpha \in(p-1,1-\varepsilon), \varepsilon \in(0,1)$. Let $I: \Omega_{p}(M) \rightarrow \Omega_{p}(N)$ be

\footnotetext{
${ }^{4}$ That is to say, for all $(x, v) \in T M, f(x, v): N \rightarrow T N$ is a vector field, moreover, $v \rightarrow f(x, v)$ is linear. 
the Itô map defined by $I(x)=y$, where y solves the differential equation on $M$ driven by a finite p-variational path $x \in \Omega_{p}(M)$ :

$$
d y_{t}=f\left(y_{t}\right) d x_{t}, \quad y_{0}=n_{0} \in N .
$$

Then $I: \Omega_{p}(M) \rightarrow \Omega_{p}(N)$ is $C^{k, \frac{\varepsilon}{1+\varepsilon}}$ in the sense of Fréchet.

Proof. - Following the Nash isometric embedding theorem, we regard $M$ and $N$ as closed sub-manifolds of some Euclidean space $\mathbb{R}^{l}$. Using the Whitney extension theorem for Lipschitz functions (see [25, p. 177]), we can extend the $\operatorname{Lip}(k+\alpha+\varepsilon)$-function $f: M \rightarrow \operatorname{End}(T M, T N)$ to a $\operatorname{Lip}(k+\alpha+\varepsilon)$-function $\bar{f}: \mathbb{R}^{l} \rightarrow \operatorname{End}\left(T \mathbb{R}^{l}, T \mathbb{R}^{l}\right)$. Let $i_{M}: M \rightarrow \mathbb{R}^{l}$ be our Nash embedding. Then $i_{M} \in C^{\infty}\left(M, \mathbb{R}^{l}\right)$. Let $\bar{I}: \Omega_{p}\left(\mathbb{R}^{l}\right) \rightarrow \Omega_{p}\left(\mathbb{R}^{l}\right)$ be the Itô map associated to the differential equation

$$
d \bar{y}=\bar{f}(y) d \bar{x}, \quad \bar{y}_{0}=a \in M .
$$

By Theorem 2.2, we have $\bar{I} \in C^{k, \frac{\varepsilon}{1+\varepsilon}}\left(\Omega_{p}\left(\mathbb{R}^{l}\right), \Omega_{p}\left(\mathbb{R}^{l}\right)\right)$. By the chain rule and the uniqueness of solutions to differential equations

$$
I=\bar{I} \circ i_{M},
$$

we can easily conclude Theorem 3.1.

Remark 3.2. - By E.M. Stein [25] the extension of the vector fields from $M$ to $\mathbb{R}^{l}$ increases the $\operatorname{Lip}(k+\alpha+\varepsilon)$ norm in a bounded way that is independent of the manifold $M$, i.e., for some constant $C_{l}$ which only depends on $M$ through $l$, we have

$$
\|\bar{f}\|_{L i p(k+\alpha+\varepsilon)} \leqslant C_{l}\|f\|_{L i p(k+\alpha+\varepsilon)} .
$$

The compactness of $N$ ensures the extrinsic and intrinsic distances on $N$ are equivalent and so also ensures that the induced $p$-variation metrics on paths in $N$ are also equivalent.

\subsection{Dynamics on path spaces}

By [17], for any $\gamma \in \Omega_{p}(M)$, we can solve the parallel transport differential equation along $\gamma$ :

$$
\nabla_{d \gamma(s)} U_{s}(\gamma)=0, \quad s \in[0,1] .
$$

Working in a local coordinate chart at $\gamma(s)$, it can be written as:

$$
d U_{s}^{k}(\gamma)=-\Gamma_{i j}^{k}(\gamma(s)) d \gamma^{i}(s) U_{s}^{j}(\gamma), \quad s \in[0,1]
$$

with the initial condition $U_{0}(\gamma)=U_{0} \in O(M)$, where $\Gamma_{i j}^{k}$ denotes the Christoffel symbol of the Levi-Civita connection, and $U_{0}$ is a given initial orthonormal frame over $\gamma(0)$. Since $U_{t}(\gamma)$ is an isometry from $T_{\gamma(0)} M$ to $T_{\gamma(t)} M$, we see that $\Omega_{p}(M)$ is a Banach manifold modelled on $\Omega_{p}\left(\mathbb{R}^{d}\right)$ with a global chart $U(\gamma)(s):=U_{s}(\gamma)$ from $\Omega_{p}\left(T_{m_{0}}(M)\right)$ to $\Omega_{p}(M), s \in[0,1]$.

Remark 3.3. - Any bounded variation path $\gamma$ is differentiable almost everywhere on $[0,1]$. So one can define $J: \Omega_{p}(M) \rightarrow \Omega_{p}\left(T_{m_{0}}(M)\right)$ by

$$
\begin{gathered}
x(t)=\int_{0}^{t} U^{-1}(s) d \gamma(s), \quad t \in[0,1] . \\
J(\gamma):=x .
\end{gathered}
$$


Now, $\gamma \rightarrow U$ is differentiable if $\Gamma_{i j}^{k}$ is $\operatorname{Lip}(p+1+\varepsilon)$. Using the smoothness of the Young integral, we see that $J$ is Fréchet differentiable. Conversely, if $x \in \Omega_{p}\left(T_{m_{0}}(M)\right)$, then there are unique $U \in \Omega_{p}(O(M))$ and $\gamma \in \Omega_{p}(M)$ satisfying (3.1) and such that

$$
d \gamma_{t}=U\left(\gamma_{t}\right) d \gamma_{t}, \quad \gamma_{0}=m, U_{0}=u_{0} .
$$

Then the Cartan-Itô development of paths in $\Omega_{p}\left(T_{m_{0}}(M)\right)$ is defined by the map

$$
\begin{gathered}
I: \Omega_{p}\left(T_{m_{0}}(M)\right) \rightarrow \Omega_{p}(M), \\
I(x):=\gamma .
\end{gathered}
$$

By Theorem 3.1, $I$ is Fréchet differentiable. Moreover, one can prove that $I \circ J=I d_{\Omega_{p}(M)}$, and $J \circ I=I d_{\Omega_{p}\left(T_{m_{0}} M\right)}$. Hence, one can deduce from the chain rule that the derivatives of $I$ and $J$ are invertible at every point.

Remark 3.4. - Let $h \in \Omega_{p}\left(T_{m_{0}} M\right)$. For any $\gamma \in \Omega_{p}(M)$ with the initial condition $\gamma(0)=m_{0}$, we define a vector field along $\gamma$ by

$$
X_{h}(\gamma, s)=U_{s}(\gamma) h(s)
$$

By Theorem 2.2, the Itô map $\gamma \rightarrow U(\gamma)$ is a local $C^{1}$-map (indeed it is a local $C^{\infty}$-smooth map). Hence $\gamma \rightarrow X_{h}(\gamma)$ is a local $C^{1}$-Lipschitz vector field on $\Omega_{p}(M)$. Therefore, applying the classical results showing the existence and the uniqueness of solutions to differential equations on Banach spaces, see e.g. [6], we have the following:

THEOREM 3.5. - The vector field $X_{h}$ generates a flow on $\Omega_{p}(M)$. More precisely, there exist some $T>0$ and a $C^{1}$-curve $\Phi:[-T, T] \times \Omega_{p}(M) \rightarrow \Omega_{p}(M)$ such that for every $\gamma \in \Omega_{p}(M)$ and $t \in[-T, T]$,

$$
\begin{aligned}
\frac{\partial}{\partial t} \Phi_{t}(\gamma) & =X_{h}\left(\Phi_{t}(\gamma)\right), \\
\Phi_{0}(\gamma) & =\gamma
\end{aligned}
$$

In view of earlier work on the Brownian setting, see e.g. [7], we call $\Phi_{t}$ the Driver flow generated by the vector field $X_{h}$ on the finite $p$-variation path space $\Omega_{p}(M)$. Here we would like to point out that the vector fields $X_{h}$ and the corresponding Driver flows are the natural ways to develop the variational calculus on the path space $\Omega_{p}(M)$ for the following reasons. First, the parallel transport $U: \Omega_{p}(M) \rightarrow T \Omega_{p}(M)$ along a curve $\gamma$ provides with us a "moving frame" which is global over $\Omega_{p}(M)$ and preserves the $p$-variation metric. Second, the parallel transport gives us a way to test whether a vector field along every $\gamma \in \Omega_{p}(M)$ is good or not. The most important point is that, since for $h \in \Omega_{p}\left(T_{m_{0}} M\right)$, the vector field $X_{h}$ generates a local flow on $\Omega_{p}(M)$,we can use this flow to introduce the variational calculus on $\Omega_{p}(M)$ in a usual way: for every functional $F$ defined on $\Omega_{p}(M)$, we say that $F$ is a differentiable functional on $\Omega_{p}(M)$ if the following limit exists

$$
D_{X_{h}} F(\gamma):=\lim _{\varepsilon \rightarrow 0} \frac{F\left(\gamma_{\varepsilon}\right)-F(\gamma)}{\varepsilon} .
$$

Remark 3.6. - In the case of smooth path spaces, the Cartan development map and its inverse, i.e., the anti-development map, were introduced by E. Cartan [2-4]. In [10], Eells and Elworthy proved that the Cartan development map and its inverse, when defined on finite energy path spaces, are diffeomorphisms of infinite dimensional Hilbert manifolds. See also Andersson and Driver [1] for a simplified proof. In [13,16], the first named author proved that the stochastic

$4^{\mathrm{e}}$ SÉRIE - TOME $39-2006-\mathrm{N}^{\circ} 4$ 
Cartan-Itô development map and its inverse (i.e., the stochastic anti-development) are smooth in the sense of stochastic variational calculus on infinite dimensional continuous path spaces. We also point out that, strictly, to talk about smooth maps one needs charts on the target space. In general charts around paths on a manifold are messy, however, one can instead use this construction with a connection to construct a chart. It is clear that if we take two connections and developing the paths out with one connection and back with the other one has a differentiable functional and so provide a consistent family of (global) charts.

\subsection{Fractional like motions on path spaces}

As our first simple application we show how it is possible to construct fractional Brownian motion type processes on path spaces. Let $\left(h_{i}\right)_{i=1}^{n}$ be a family of elements of $\Omega_{p}\left(T_{m_{0}} M\right)$. Take $w_{t}$ to be $n$-dimensional fractional Brownian motion with the Hurst parameter $H \in(1 / 4,1)$; by [5] we can consider $w$ as a $q$-rough path for any $q>H^{-1}$. Consider the stochastic differential equation on the path space $\Omega_{p}(M)$ driven by $w$ and starting from a given $p$-path $\gamma$ in $M$ as follows:

$$
\begin{aligned}
d \Pi_{t} & =\sum_{i=1}^{n} X_{h_{i}}\left(\Pi_{t}\right) \circ d w_{t}^{i}, \\
\Pi_{0} & =\gamma
\end{aligned}
$$

Since $p \in[1,2)$, we can apply our main theorems Theorem 2.2 and Theorem 3.1 to conclude that the vector fields $X_{h_{i}}$ are locally $C^{\infty}$ on $\Omega_{p}(M)$. Therefore, applying the standard theory of differential equations driven by $q$-rough paths (which is infinite dimensional but requires smoothness of the vector fields) [17], one sees that Eq. (3.4) has a unique solution $\Pi_{t}$ which is continuously dependent on the initial path $\gamma$ and the driving path $w$.

Of course $\Pi_{t}$ is a nonlinear object and does not the scaling or invariance properties that characterise true fractional Brownian motion and there is a great deal of arbitrariness in the choices of $h_{i}$ in $\Omega_{p}\left(T_{m_{0}} M\right)$.

\subsection{Infinite dimensional Brownian motions on path spaces}

As a second application of our methods we specialise the above example to the case where $H=1 / 2$ but extend the analysis to the case where the dimension of the driving Brownian motion is infinite. We construct an infinite dimensional Brownian motion on path spaces.

Fix a control $\omega$ on the triangle $\Delta=\{(s, t): 0 \leqslant s \leqslant t \leqslant 1\}$. Take $p \in[1,2)$ and $p^{\prime} \in(p, 2)$. Let $E$ be the completion of $\Omega_{p, \omega}\left([0,1], \mathbb{R}^{d}\right)$ with respect to $\|\cdot\|_{p^{\prime}}$. The smooth paths are dense in $E$ with respect to $\|\cdot\|_{p^{\prime}}$ and so $E$ is a separable Banach space. Let $\left\{e_{n}, n \in \mathbb{N}\right\}$ be a countable collection of linearly independent elements in the unit ball of $E$ such that the vector space spanned by $\left(e_{n}\right)$ is dense in $E$. Let $\left\{x_{n}(t), n \in \mathbb{N}\right\}$ be a sequence of independent Brownian motions; then $\sum_{n=1}^{\infty} n^{-2} x_{n} e_{n}$ converges in probability to an $E$-valued Brownian denoted by $B_{t}$ with law $\mu_{t}$ at time $t$. Then the support of $\mu=\mu_{1}$ is dense in $\operatorname{span}\left(e_{n}\right)$ and so is in $E$. Let $H:=\left\{h=\sum_{n=1}^{\infty} a_{n} e_{n}:\|h\|_{H}<\infty\right\}$ where $\|h\|_{H}^{2}:=\sum_{n=1}^{\infty} a_{n}^{2} n^{4}$. Then

$$
\left\|\sum_{n=1}^{\infty} a_{n} e_{n}\right\|_{E} \leqslant \sum_{n=1}^{\infty}\left|a_{n}\right| \leqslant\left(\sum_{n=1}^{\infty} a_{n}^{2} n^{4}\right)^{1 / 2}\left(\sum_{n=1}^{\infty} n^{-4}\right)^{1 / 2}
$$

and $\|h\|_{E} \leqslant C\|h\|_{H}$. So $H$ is densely embedded in $E$ and $(E, H, \mu)$ is a Wiener space in the sense of Leonard Gross. 
Let $\left\{h_{n}, n \in H\right\}$ be an orthonormal basis of $H$. Then $h_{n} \in E \subset \Omega_{p}\left([0,1], T_{m_{0}} M\right)$. Using Theorem 3.1 we can see that the vector fields $X_{h_{i}}$ defined in (3.3) are $C^{2}$ in the injective tensor product norm as a map

$$
\Omega_{p}(M) \stackrel{X_{h_{i}}}{\longrightarrow} \Omega_{p}(T M)
$$

Define

$$
X: \Omega_{p}\left(T_{m_{0}} M\right) \times \Omega_{p}(M) \rightarrow \Omega_{p}(T M)
$$

by

$$
(h, \gamma) \rightarrow X_{h}(\gamma) .
$$

Then $X$ is a bounded linear operator from $E$ to the $C^{2}$ vector fields on $\Omega_{p}(M)$. Since every Brownian Motion on a Banach space is a rough path when one uses the injective tensor product [19] we have the following

THEOREM 3.7. - Let $p \in[1,2)$. For any $\gamma \in \Omega_{p}(M)$ with $\gamma(0)=m_{0}$, there exists a unique diffusion process $5{ }^{5}\{t \in[-T, T]\}$ such that

$$
\begin{aligned}
d \Sigma_{t} & =X\left(\Sigma_{t}\right) \circ d B_{t}, \\
\Sigma_{0} & =\gamma .
\end{aligned}
$$

Following [23,8], we call $\left\{\Sigma_{t}, t \in[0, T]\right\}$ a Brownian motion on the path space $\Omega_{p}(M)$ and refer to the law $\Sigma_{t}$ as the heat kernel measure on $\Omega_{p}(M)$ and the law of $\Sigma$ as the associated Wiener measure on $C\left([0, T], \Omega_{p}(M)\right)$. Of course, the above construction only depends on the reproducing Hilbert space $H$ of the Gaussian space $(E, H, \mu)$ (and this was somewhat arbitrarily chosen) but is at least independent of the choice of $\left\{h_{n}, n \in \mathbb{N}\right\}$. It might be interesting to investigate the construction for other Gaussian measures on $E$.

\section{REFERENCES}

[1] Andersson L., Driver B.K., Finite-dimensional approximations to Wiener measure and path integral formulas on manifolds, J. Funct. Anal. 165 (2) (1999) 430-498.

[2] CARTAn E., Leçons sur la géométrie projective complexe, Les Grands Classiques Gauthier-Villars, 1931.

[3] CARTAn E., La théorie des groupes finis et continus et la géométrie différentielle traitées par la méthode du repère mobile, Les Grands Classiques Gauthier-Villars, 1937.

[4] CARTAN E., Leçons sur la théorie des espaces à connexion projective, Les Grands Classiques Gauthier-Villars, 1937.

[5] Coutin L., QIAN Z., Stochastic analysis, rough path analysis and fractional Brownian motions, Probab. Theory Related Fields 122 (1) (2002) 108-140.

[6] Dieudonné J., Foundations of Mordern Analysis, vol. I, Academic Press, New York, 1969.

[7] Driver B.K., A Cameron-Martin type quasi-invariance theorem for Brownian motion on a compact manifold, J. Funct. Anal. 109 (1992) 272-376.

[8] DRIVER B.K., Integration by parts and quasi-invariance for heat kernel measures on loop groups, J. Funct. Anal. 149 (2) (1997) 470-547.

[9] Dudley R.M., Norvaisa R., Differentiability of Six Operators on Nonsmooth Functions and $p$-Variation, Lecture Notes in Math., vol. 1703, Springer, Berlin, 1999.

\footnotetext{
${ }^{5}$ Here the "diffusion process" means that the trajectories $t \rightarrow \Sigma_{t}$ are almost surely $2+\epsilon$-rough paths for any arbitrary small $\epsilon>0$, which corresponds to the classical situation of diffusion processes on any finite dimensional Euclidean space and manifold. See e.g. [18].

$4^{\mathrm{e}}$ SÉRIE - TOME $39-2006-\mathrm{N}^{\circ} 4$
} 
[10] Eells J., Elworthy K.D., Wiener integration on certain manifolds, in: Problems in Non-Linear Analysis (C.I.M.E., IV Ciclo, Varenna, 1970), Edizioni Cremonese, Rome, 1971, pp. 67-94.

[11] Itô K., The Brownian motion and tensor fields on Riemannian manifold, in: International Congress of Mathematicians 1963 (Stocholm), Inst. Mittag-Leffler, Djursholm, pp. 536-539.

[12] Klingenberg W., Lectures on Closed Geodesics, Springer, Berlin, 1978.

[13] LI X.D., Stochastic analysis and geometry on path and loop spaces, PhD thesis, Academia Sinica (Beijing) and University of Lisbon, 1999.

[14] LI X.D., Existence and uniqueness of geodesics on path spaces, J. Funct. Anal. 173 (2000) 182-202.

[15] LI X.D., Connections, geodesics and measures on path and loop spaces, Preprint, 2002.

[16] LI X.D., Sobolev spaces and capacities theory on path spaces over a compact Riemannian manifold, Probab. Theory Related Fields 125 (1) (2003) 96-134.

[17] LyONS T., Differential equations driven by rough signals. I. An extension of an inequality of L.C. Young, Math. Res. Lett. 1 (4) (1994) 451-464.

[18] LyONS T., Differential equations driven by rough signals, Revista Matemática Iberoamericana 14 (2) (1998) 215-300.

[19] Ledoux M., Lyons T., Qian Z., Lévy area of Wiener processes in Banach spaces, Ann. Probab. 30 (2) (2002) 546-578.

[20] Lyons T., Qian Z., A class of vector fields on path space, J. Funct. Anal. 145 (1) (1997) 205-223.

[21] LyONS T., QIAN Z., Stochastic Jacobi fields and vector fields induced by varying area on path spaces, Probab. Theory Related Fields 109 (4) (1997) 539-570.

[22] LyOns T., Qian Z., System Control and Rough Paths, Oxford Mathematical Monographs, Clarendon Press, Oxford, 2002.

[23] Malliavin P., Hypoellipticity in infinite dimensions, in: Diffusion processes and related problems in analysis, vol. I (Evanston, IL, 1989), Birkhäuser Boston, 1990, pp. 17-31.

[24] Malliavin P., Stochastic Analysis, Springer, Berlin, 1997.

[25] STEIN E.M., Singular Integrals and Differentiability Properties of Functions, Princeton Mathematical Series, vol. 30, Princeton University Press, Princeton, NJ, 1970.

(Manuscrit reçu le 19 août 2005 ; accepté, après révision, le 30 mai 2006.)

\footnotetext{
Xiang-Dong LI

Mathematical Institute,

University of Oxford,

24-29, St. Giles',

Oxford, OX1 3LB, UK

Current address:

Institut de Mathématiques,

Université Paul Sabatier,

118, route de Narbonne,

31062, Toulouse, France

E-mail: xiang@cict.fr
}

Terry J. LYONS

Mathematical Institute,

University of Oxford,

24-29, St. Giles',

Oxford, OX1 3LB, UK

E-mail: tlyons@maths.ox.ac.uk 